Research Article

\title{
Efficient Estimation of the Generalized Quasi-Lindley Distribution Parameters under Ranked Set Sampling and Applications
}

\author{
Amer Ibrahim Al-Omari ${ }^{D},{ }^{1}$ SidAhmed Benchiha ${ }^{(D)},{ }^{2}$ and Ibrahim M. Almanjahie $\mathbb{D}^{3,4}$ \\ ${ }^{1}$ Department of Mathematics, Faculty of Science, Al al-Bayt University, Mafraq, Jordan \\ ${ }^{2}$ Department of Mathematics, University of Jordan, Amman, Jordan \\ ${ }^{3}$ Department of Mathematics, College of Science, King Khalid University, Abha 62529, Saudi Arabia \\ ${ }^{4}$ Statistical Research and Studies Support Unit, King Khalid University, Abha 62529, Saudi Arabia \\ Correspondence should be addressed to Ibrahim M. Almanjahie; imalmanjahi@kku.edu.sa
}

Received 12 April 2021; Revised 30 June 2021; Accepted 9 July 2021; Published 21 July 2021

Academic Editor: Ahmed Salem

Copyright (C) 2021 Amer Ibrahim Al-Omari et al. This is an open access article distributed under the Creative Commons Attribution License, which permits unrestricted use, distribution, and reproduction in any medium, provided the original work is properly cited.

\begin{abstract}
Ranked set sampling is a very useful method to collect data when the actual measurement of the units in a population is difficult or expensive. Recently, the generalized quasi-Lindley distribution is suggested as a new continuous lifetime distribution. In this article, the ranked set sampling method is considered to estimate the parameters of the generalized quasi-Lindley distribution. Several estimation methods are used, including the maximum likelihood, the maximum product of spacings, ordinary least squares, weighted least squares, Cramer-von Mises, and Anderson-Darling methods. The performance of the proposed ranked set sampling based estimators is achieved through a simulation study in terms of bias and mean squared errors compared to the simple random sample. Additional results are obtained based on real data for the survival times of 72 guinea pigs and 23 ball bearings. The simulation study results and the real data applications showed the superiority of the proposed ranked set sampling estimators compared to the simple random sample competitors based on the same number of measuring units.
\end{abstract}

\section{Introduction}

One of the significant interesting fields in statistics is the cost-effective sampling methods. The motivation of this field arises from its superiority in facilitating data collection, especially when collecting data of interest consumes a long time or is expensive. Over the past decades, researchers developed different sampling methods in order to achieve reliable results with low cost and more accuracy. McIntyre [1] proposed a new sampling method for estimating the mean of pasture and forage yields in Australia. This method is known as ranked set sampling (RSS) and has greater efficiency than the commonly used simple random sampling (SRS). Later, Halls and Dell [2]; Takahasi and Wakimoto [3]; and Dell and Clutter [4] published others studies on the RSS method. Due to its cost-effectiveness, it is used in wide applications, including reliability, estimation of population parameters, statistical quality control, medicine, acceptance sampling plans, and so on (see Chen et al. [5-7], Haq et al. [8], and Al-Omari and Haq $[9,10])$.

It is well documented that the RSS method is an attractive procedure and more acclimated to the nature of the underlying data. This advantage motivated researchers to propose and study new RSS schemes (see, for example, the double ranked set by Al-Saleh and Al-Kadiri [11]; median ranked set sampling by Mutllak [12]; neoteric ranked set sampling by Zamanzade and Al-Omari [13]; extreme ranked set sampling by Samawi et al. [14]; and L ranked set sampling by Al-Nasser [15]). For a good review of RSS and more motivations on this method, one can refer to Al-Omari and Bouza [16]; Al-Hadhrami and Al-Omari [17]; Haq et al. [18]; Santiago et al. [19]; and Haq et al. [20]. 
The problem of estimating distributions parameters, in general, is considered by many authors. For example, Nassar et al. [21] considered parameters estimation of the new extension of Weibull distribution. Nassar et al. [22] treated the estimation problem using the alpha power exponential distribution. Later, Afify and Mohamed [23] and Afifiy et al. [25] dealt with parameter estimation based on the new three-parameter exponential distribution and the Weibull Marshall -Olkin Lindley distribution, respectively. Recently, Alfaer et al. (2021) considered the extended log-logistic distribution and estimated its parameters. Although these works considered parameter estimations in related distributions, their works did not deal with sampling design techniques.

Undoubtedly, the parametric estimation method using the sampling design technique plays a vital role in statistical inference. Many studies considered the estimation of parameters based on RSS designs and their extensions using different estimation methods. Yousef and Al-Subh [26] estimated the Gumbel parameters using the maximum likelihood method, method of moment, and the method of regression. Hussian [27] used the Bayesian and maximum likelihood estimation methods to estimate the Kumaraswamy distribution parameters. Chen et al. [28] estimated the scale parameter for the scale distribution using moving extreme ranked set sampling, and Abu-Dayyeh et al. [29] considered the logistic method for parameter estimation based on both SRS and RSS. Pedroso et al. [30] considered the RSS to estimate the parameters of the twoparameter Birnbaum-Saunders distribution, and Akgul et al. [31] used the same RSS in system reliability estimation for generalized inverse Lindley distribution. (see also Taconeli and Bonat [32] for some estimation methods based on the RSS). For more details on parameter estimation, the readers are referred to Stokes [33]; Dey et al. [34]; Khamnei and Mayan [35]; and AlSaleh and Al-Hadhrami [36].
This paper aims to study the performance of using RSS design in estimating the parameters of the generalized quasiLindley distribution (GQLD) introduced recently by Benchiha and Al-Omari [37]. A random variable $X$ is said to follow a GQLD distribution with parameters $\theta$ and $\alpha$ if its pdf is given by

$$
\begin{gathered}
f(x ; \alpha, \theta)=\frac{\theta^{2}\left(\left(\theta^{2} x^{3} / 6\right)+\alpha \theta x^{2}+\alpha^{2} x\right) e^{-\theta x}}{(\alpha+1)^{2}} \\
x \geq 0, \alpha>-1, \theta \geq 0 .
\end{gathered}
$$

with cdf given by

$$
F(x ; \alpha, \theta)=1-\frac{\left(\theta^{3} x^{3}+3(2 \alpha+1) \theta^{2} x^{2}+6(\alpha+1)^{2}(\theta x+1)\right) e^{-\theta x}}{6(\alpha+1)^{2}} .
$$

The first two moments of $X$, respectively, are

$$
\begin{aligned}
E(X) & =\frac{6\left(\theta^{2}+1\right)+6 \theta+6}{3(\theta+1)^{2} \alpha}, \\
E\left(X^{2}\right) & =\frac{6(\theta+1)^{2}+2(6 \theta+5)+4}{(\theta+1)^{2} \alpha^{2}} .
\end{aligned}
$$

The variance of the GQLD distribution is given by

$$
V(X)=E\left(X^{2}\right)-(E(X))^{2}=\frac{2\left(\theta^{2}+4 \theta+2\right)}{(\theta+1)^{2} \alpha^{2}} .
$$

The corresponding reliability and hazard functions of the GQLD distribution, for $x>0, \theta>-1, \alpha>0$, are given, respectively, by

$$
\begin{aligned}
& R_{\mathrm{GQLD}}(x ; \alpha, \theta)=\frac{\left(\alpha^{3} x^{3}+3(2 \theta+1) \alpha^{2} x^{2}+6(\theta+1)^{2} \alpha x+6(\theta+1)^{2}\right) \mathrm{e}^{-\alpha x}}{6(\theta+1)^{2}} \\
& H_{\mathrm{GQLD}}(x ; \alpha, \theta)=\frac{6 \alpha^{2}\left(\left(\alpha^{2} x^{3} / 6\right)+\theta \alpha x^{2}+\theta^{2} x\right)}{\alpha^{3} x^{3}+3(2 \theta+1) \alpha^{2} x^{2}+6(\theta+1)^{2} \alpha x+6(\theta+1)^{2}}
\end{aligned}
$$

To the best of our knowledge, there are no published papers which used the RSS in estimating the parameters of GQLD. The remainder of this paper is organized as follows. The RSS method is explained and the suggested various estimators for the GQLD are given in Section 2. In Section 3, a simulation study is provided to investigate the performance of the RSS estimators relative to the SRS counterparts based on the same number of measured units. Applications to real datasets fitted to the GQLD are given in Section 4. The paper is ended in Section 5 with concluding remarks and suggestions for future works.

\section{Methods of Estimation}

In this section, six methods of estimation are considered for estimating the unknown parameters $\theta$ and $\alpha$ of the GQLD distribution using RSS design. These methods are the maximum likelihood method, method of maximum product of spacings, ordinary least squares method, weighted least squares method, Cramer-von Mises method, and Anderson-Darling method. The RSS strategy can be described as follows:

(1) Select a simple random sample of size $k^{2}$ units from the desired population. Randomly partition them into $k$ sets of each size $k$, where $k$ is known as the set size.

(2) Rank the units within each set of size $k$ from smallest to largest with respect to the variable of interest.

(3) Obtain the $i$ th order statistic from the $i$ th set, for $i=1,2, \ldots, k$. 
(4) Repeat Steps (1)-(3), $n$ times (cycles) if needed, to get a ranked set sample of size $N=n k$.

The resulting sample is denoted as $\left\{X_{[i] j}, i=\right.$ $1,2, \ldots, k ; j=1,2, \ldots, n\}$, where $X_{[i] j}$ is the $i$ th largest unit in a set of size $k$ in the $j$ th cycle. It is of interest to note that perfect ranking is assumed in this study.

2.1. Maximum Likelihood Estimation. Let $\left\{X_{[i] j}, i=\right.$ $1,2, \ldots, k ; j=1,2, \ldots, r\}$ denote the $i$ th order statistics from the $i$ th set of size $k$ at the $j$ th cycle, and take it as the RSS data for $X$ of a sample size $n=k r$. Then, the maximum likelihood function based on the RSS sample is given by

$$
L_{\mathrm{RSS}}(\alpha, \theta)=\prod_{j=1}^{r} \prod_{i=1}^{k} f_{(i: k)}\left(x_{[i] j}, \alpha, \theta\right),
$$

where

$$
\begin{aligned}
f_{(i: k)}\left(x_{[i] j}, \alpha, \theta\right)= & \frac{k !}{(i-1) !(k-i) !}\left[F\left(x_{[i] j}\right)\right]^{i-1}\left[1-F\left(x_{[i] j}\right)\right]^{k-i} f\left(x_{[i] j}\right) \\
= & \frac{k !}{(i-1) !(k-i) !}\left[1-\frac{\left(\theta^{3} x^{3}+3(2 \alpha+1) \theta^{2} x^{2}+6(\alpha+1)^{2}(\theta x+1)\right) e^{-\theta x}}{6(\alpha+1)^{2}}\right]^{i-1} \\
& \times\left[\frac{\left(\theta^{3} x^{3}+3(2 \alpha+1) \theta^{2} x^{2}+6(\alpha+1)^{2}(\theta x+1)\right) e^{-\theta x}}{6(\alpha+1)^{2}}\right]^{k-i} \\
& \times\left(\frac{\theta^{2}\left(\left(\theta^{2} x^{3} / 6\right)+\alpha \theta x^{2}+\alpha^{2} x\right) e^{-\theta x}}{(\alpha+1)^{2}}\right) .
\end{aligned}
$$

The log-likelihood function, $\mathfrak{R}_{\mathrm{RSS}}=\ln L_{\mathrm{RSS}}(\alpha, \theta)$, is

$$
\begin{aligned}
\mathfrak{R}_{\mathrm{RSS}}= & \sum_{j=1}^{r} \sum_{i=1}^{k} \log \left\{f_{i: k}\left(x_{[i] j}, \theta, \alpha\right)\right\} \\
= & \sum_{j}^{r} \sum_{i=1}^{k} \log \left(\frac{k !}{(i-1) !(k-i) !}\right)+\sum_{j=1}^{r} \sum_{i=1}^{k}(i-1) \log \left(F\left(x_{[i] j}\right)\right) \\
& +\sum_{j=1}^{r} \sum_{i=1}^{k}(k-i) \log \left(1-F\left(x_{[i] j}\right)\right)+\sum_{j=1}^{r} \sum_{i=1}^{k} \log f\left(x_{[i] j}\right) .
\end{aligned}
$$

The estimators of $\alpha$ and $\theta$ of the GQLD using RSS can be obtained by solving the nonlinear equations

$$
\begin{aligned}
& \frac{\partial \Re_{\mathrm{RSS}}}{\partial \theta}=0, \\
& \frac{\partial \mathfrak{R}_{\mathrm{RSS}}}{\partial \alpha}=0 .
\end{aligned}
$$

2.2. Method of Maximum Product of Spacings. Cheng and Amin $[38,39]$ introduced the method of maximum product of spacings (MPS). This method is based on maximization of the geometric mean of spacings in the data. The MPS is a consistent and efficient in most general cases. Consider the ordered units $y_{(1: N)}, y_{(2: N)}, \ldots, y_{(N: N)}$ that form a ranked set sample of size $N=n k$ where $n$ is the number of cycle and $k$ is the set size from the GQLD. Then, the uniform spacing is given by

$$
D_{i}(\alpha, \theta)=F\left(y_{(i: N)} \mid \alpha, \theta\right)-F\left(y_{(i-1: N)} \mid \alpha, \theta\right), \quad i=1,2, \ldots, N,
$$

where $F\left(y_{(0: N)} \mid \alpha, \theta\right)=0$ and $F\left(y_{(N+1: N)} \mid \alpha, \theta\right)=1$. Clearly, $\sum_{i=1}^{N+1} D_{i}(\alpha, \theta)=1$.

The MPS estimators, $\widehat{\alpha}_{\mathrm{MPS}}$ and $\widehat{\theta}_{\mathrm{MPS}}$, are the estimates of $\alpha$ and $\theta$, which maximize the geometric mean of the spacing $H(\alpha, \theta \mid y)$, where

$$
H(\alpha, \theta \mid y)=\left[\prod_{i=1}^{N+1} D_{i}(\alpha, \theta)\right]^{1 /(N+1)} .
$$

The natural logarithm of (11) is

$$
M(\alpha, \theta \mid y)=\frac{1}{N+1} \sum_{i=1}^{N+1} \log D_{i}(\alpha, \theta) .
$$

The estimators $\widehat{\alpha}_{\text {MPS }}$ and $\widehat{\theta}_{\text {MPS }}$ of the parameters $\alpha$ and $\theta$, respectively, can also be obtained by solving the nonlinear equations: 


$$
\begin{aligned}
& \frac{\partial}{\partial \alpha} M(\alpha, \theta)=\frac{1}{N+1} \sum_{i=1}^{N+1} \frac{1}{D_{i}(\alpha, \theta)}\left[\Psi_{1}\left(y_{(i: N)} \mid \alpha, \theta\right)-\Psi_{1}\left(y_{(i-1: N)} \mid \alpha, \theta\right)\right]=0 \\
& \frac{\partial}{\partial \theta} M(\alpha, \theta)=\frac{1}{N+1} \sum_{i=1}^{N+1} \frac{1}{D_{i}(\alpha, \theta)}\left[\Psi_{2}\left(y_{(i: N)} \mid \alpha, \theta\right)-\Psi_{2}\left(y_{(i-1: N)} \mid \alpha, \theta\right)\right]=0
\end{aligned}
$$

where

$$
\begin{aligned}
\Psi_{1}\left(y_{(i: N)} \mid \alpha, \theta\right) & =\frac{\partial}{\partial \alpha} F\left(y_{(i: N)} \mid \alpha, \theta\right) \\
& =\frac{\theta^{2} y_{(i: N)}^{2} e^{-\theta y_{(i: N)}}\left(3 \alpha+\theta y_{(i: N)}\right)}{3(\alpha+1)^{3}}
\end{aligned}
$$

and

$$
\begin{aligned}
\Psi_{2}\left(y_{(i: N)} \mid \alpha, \theta\right) & =\frac{\partial}{\partial \theta} F\left(y_{(i: N)} \mid \alpha, \theta\right) \\
& =\frac{y_{(i: N)}^{2} \theta\left(y_{(i: N)}^{2} \theta^{2}+6 \alpha y_{(i: N)} \theta+6 \alpha^{2}\right) e^{-y_{(i: N)} \theta}}{6(\alpha+1)^{2}}
\end{aligned}
$$

can be then obtained numerically.

2.3. Methods of Least Squares. Swain et al. [40] was the first who used the method of least squares to estimate the parameters of beta distribution based on one of the famous results in probability theory which indicates that $F\left(X_{(i: N)}\right) \sim \operatorname{Beta}(i, N-i+1)$ where $F$ is a cumulative distribution function and $x_{(i: N)}$ is the $i$ th order statistic of the random sample $\left(x_{1}, x_{2}, \ldots, x_{N}\right)$. Therefore, in our case, we have

$$
\begin{aligned}
\mathbb{E}\left[F\left(x_{(i: N)}\right)\right] & =\frac{i}{N+1}, \\
\operatorname{Var}\left[F\left(x_{(i: N)}\right)\right] & =\frac{i(N-i+1)}{(N+1)^{2}(N+2)} .
\end{aligned}
$$

Using the above expectations and variances, we obtain two variants of the least squares methods.

2.3.1. Ordinary Least Squares. Let the ordered units $y_{(1: N)}, y_{(2: N)}, \ldots, y_{(N: N)}$ constitute a ranked set sample of size $N=n k$. Then, the ordinary least squares (OLS) estimators, say $\widehat{\alpha}_{\text {OLS }}$ and $\widehat{\theta}_{\text {OLS }}$ of the parameters $\alpha$ and $\theta$, respectively, can be obtained by minimizing the function:

$$
\begin{aligned}
\Omega(\alpha, \theta \mid y) & =\sum_{i=1}^{N}\left[F\left(y_{(i: N)} \mid \alpha, \theta\right)-\frac{i}{N+1}\right]^{2} \\
& =\sum_{i=1}^{N}\left[1-\frac{\left(y_{(i: N)}^{3} \theta^{3}+3(2 \alpha+1) y_{(i: N)}^{2} \theta^{2}+6(\alpha+1)^{2}\left(y_{(i: N)} \theta+1\right)\right) e^{-y_{(i: N)} \theta}}{6(\alpha+1)^{2}}-\frac{i}{N+1}\right]^{2},
\end{aligned}
$$

with respect to $\alpha$ and $\theta$. Alternatively, these estimates can also be obtained by solving the following nonlinear equations:

$$
\begin{aligned}
& \sum_{i=1}^{N}\left[F\left(y_{(i: N)} \mid \alpha, \theta\right)-\frac{i}{N+1}\right] \Psi_{1}\left(y_{(i: N)} \mid \alpha, \theta\right)=0, \\
& \sum_{i=1}^{N}\left[F\left(y_{(i: N)} \mid \alpha, \theta\right)-\frac{i}{N+1}\right] \Psi_{2}\left(y_{(i: N)} \mid \alpha, \theta\right)=0,
\end{aligned}
$$

where $\Psi_{1}\left(y_{(i: N)} \mid \alpha, \theta\right)$ and $\Psi_{2}\left(y_{(i: N)} \mid \alpha, \theta\right)$ are defined as in (14) and (15), respectively.

2.3.2. Weighted Least Squares. Consider the RSS units $y_{(1: N)}, y_{(2: N)}, \ldots, y_{(N: N)}$ that form ranked set sampling of size $N=n k$. Then, the weighted least squares (WLS) estimators of $\alpha$ and $\theta$, say $\widehat{\alpha}_{\mathrm{WLS}}$ and $\widehat{\theta}_{\mathrm{WLS}}$, respectively, can be obtained by minimizing the following function: 


$$
\begin{aligned}
W(\alpha, \theta \mid y) & =\sum_{i=1}^{N} \frac{(N+1)^{2}(N+2)}{i(N-i+1)}\left[F\left(y_{(i: N)} \mid \alpha, \theta\right)-\frac{i}{N+1}\right]^{2} \\
& =\sum_{i=1}^{N} \frac{(N+1)^{2}(N+2)}{i(N-i+1)}\left[1-\frac{\left(y_{(i: N)}^{3} \theta^{3}+3(2 \alpha+1) y_{(i: N)}^{2} \theta^{2}+6(\alpha+1)^{2} y_{(i: N)} \theta+6(\alpha+1)^{2}\right) e^{-y_{(i: N)} \theta}}{6(\alpha+1)^{2}}-\frac{i}{N+1}\right]^{2}
\end{aligned}
$$

with respect to $\alpha$ and $\theta$. Equivalently, the estimates are the solution of the following nonlinear equations:

$$
\begin{aligned}
& \sum_{i=1}^{N} \frac{(N+1)^{2}(N+2)}{i(N-i+1)}\left[F\left(y_{(i: N)} \mid \alpha, \theta\right)-\frac{i}{N+1}\right], \\
& \Psi_{1}\left(y_{(i: N)} \mid \alpha, \theta\right)=0, \\
& \sum_{i=1}^{N} \frac{(N+1)^{2}(N+2)}{i(N-i+1)}\left[F\left(y_{(i: N)} \mid \alpha, \theta\right)-\frac{i}{N+1}\right], \\
& \Psi_{2}\left(y_{(i: N)} \mid \alpha, \theta\right)=0,
\end{aligned}
$$

where $\Psi_{1}\left(y_{(i: N)} \mid \alpha, \theta\right)$ and $\Psi_{2}\left(y_{(i: N)} \mid \alpha, \theta\right)$ are specified as in (14) and (15), respectively.
2.4. Methods of Minimum Distances. Methods of estimation based on minimizing some famous goodness of fit statistics are useful in many cases and give good results. Here, two popular methods based on the minimization of test statistics between the theoretical and empirical cumulative distribution functions are considered. The methods are the Cramer-von Mises method and Anderson-Darling method (for more details, see D'Agostino and Stephens [41] and Luceño [42]).

2.4.1. Cramer-von Mises Method. Consider the ordered units $y_{(1: N)}, y_{(2: N)}, \ldots, y_{(N: N)}$ that form a ranked set sample of size $N=n k$. Then, the Cramer-von Mises estimators (CV) $\widehat{\alpha}$ and $\hat{\theta}$ of $\alpha$ and $\theta$, respectively, are obtained by minimizing the function

$$
\begin{aligned}
\mathrm{CV}(\alpha, \theta) & =\frac{1}{12 N}+\sum_{i=1}^{N} \frac{(N+1)^{2}(N+2)}{i(N-i+1)}\left[F\left(y_{(i: N)} ; \alpha, \theta\right)-\frac{2 i-1}{2 N}\right]^{2} \\
& =\frac{1}{12 N}+\sum_{i=1}^{N} \frac{(N+1)^{2}(N+2)}{i(N-i+1)}\left[1-\frac{\left(\theta^{3} y_{(i: N)}^{3}+3(2 \alpha+1) \theta^{2} y_{(i: N)}^{2}+6(\alpha+1)^{2} \theta y_{(i: N)}+6(\alpha+1)^{2}\right) \mathrm{e}^{-\theta y_{(i: N)}}}{6(\alpha+1)^{2}}-\frac{2 i-1}{2 N}\right]^{2}
\end{aligned}
$$

with respect to $\alpha$ and $\theta$. Equivalently, the estimates are the solution of the following nonlinear equations:

$$
\begin{aligned}
& \sum_{i=1}^{N} \frac{(N+1)^{2}(N+2)}{i(N-i+1)}\left[F\left(y_{(i: N)} \mid \alpha, \theta\right)-\frac{2 i-1}{2 N}\right], \\
& \Psi_{1}\left(y_{(i: N)} \mid \alpha, \theta\right)=0 \\
& \sum_{i=1}^{N} \frac{(N+1)^{2}(N+2)}{i(N-i+1)}\left[F\left(y_{(i: N)} \mid \alpha, \theta\right)-\frac{2 i-1}{2 N}\right], \\
& \Psi_{2}\left(y_{(i: N)} \mid \alpha, \theta\right)=0
\end{aligned}
$$

where $\Psi_{1}\left(y_{(i: N)} \mid \alpha, \theta\right)$ and $\Psi_{2}\left(y_{(i: N)} \mid \alpha, \theta\right)$ are given in (14) and (15), respectively.
2.4.2. Anderson-Darling Method. Suppose that $y_{(1: N)}$, $y_{(2: N)}, \ldots, y_{(N: N)}$ is a ranked set sample of size $N=n k$. Then, the estimates based on the Anderson-Darling (AD) method for the GQLD distribution parameters $\alpha$ and $\theta$, denoted by $\widehat{\alpha}_{\mathrm{AD}}$ and $\widehat{\theta}_{\mathrm{AD}}$, can be obtained by minimizing the function

$$
\begin{aligned}
A(\alpha, \theta)= & -N-\frac{1}{N} \sum_{i=1}^{N}(2 i-1)\left\{\log F\left(y_{(i: N)} \mid \alpha, \theta\right)\right. \\
& \left.+\log \bar{F}\left(y_{(N-i+1: N)} \mid \alpha, \theta\right)\right\},
\end{aligned}
$$

with respect to $\alpha$ and $\theta$, or equivalently by solving the following two equations: 


$$
\begin{aligned}
& \frac{\partial A(\alpha, \theta)}{\partial \alpha}=\sum_{i=1}^{N}(2 i-1)\left\{\frac{\Psi_{1}\left(y_{(i: N)} \mid \alpha, \theta\right)}{F\left(y_{(i: N)} \mid \alpha, \theta\right)}-\frac{\Psi_{1}\left(y_{(N-i+1: N)} \mid \alpha, \theta\right)}{\bar{F}\left(y_{(N-i+1: N)} \mid \alpha, \theta\right)}\right\}=0, \\
& \frac{\partial A(\alpha, \theta)}{\partial \alpha}=\sum_{i=1}^{N}(2 i-1)\left\{\frac{\Psi_{2}\left(y_{(i: N)} \mid \alpha, \theta\right)}{F\left(y_{(i: N)} \mid \alpha, \theta\right)}-\frac{\Psi_{2}\left(y_{(N-i+1: N)} \mid \alpha, \theta\right)}{\bar{F}\left(y_{(N-i+1: N)} \mid \alpha, \theta\right)}\right\}=0,
\end{aligned}
$$

where $\Psi_{1}\left(y_{(i: N)} \mid \alpha, \theta\right)$ and $\Psi_{2}\left(y_{(i: N)} \mid \alpha, \theta\right)$ are as specified in (14) and (15), respectively.

\section{Simulation}

In order to evaluate the performance of the estimation methods under RSS, a simulation study is conducted by using $\mathrm{R}$ software. 1000 samples are generated from the GQLD with different parameters values as $(1,1),(1,3)$, $(0.5,1)$, and $(0.8,1.5)$ in different sizes for both RSS and SRS. For the RSS design, the number of cycles is selected to be $n=3,4$, and 5 while the set size $k$ is taken as 5,10 , and 15 . In each case, we combine $n$ with all values of $k$ to study the effect of set size and the number of cycles. For the SRS design, the size of SRS is $N=n k$ which is required for having the same size in each design. We have considered perfect ranking assumptions. The mean squared error (MSE) is calculated for each estimator in order to compare SRS and RSS. The MSE and the efficiency (Eff) are calculated by

$$
\begin{aligned}
\operatorname{MSE}_{\widehat{\alpha}} & =\frac{1}{1000} \sum_{i=1}^{1000}(\widehat{\alpha}-\alpha)^{2}, \\
\operatorname{MSE}_{\theta} & =\frac{1}{1000} \sum_{i=1}^{1000}(\widehat{\theta}-\theta)^{2}, \\
\operatorname{Eff}_{\widehat{\alpha}}\left(\widehat{\alpha}_{\mathrm{RSS}}, \widehat{\alpha}_{\mathrm{SRS}}\right) & =\frac{\operatorname{MSE}_{\widehat{\alpha}_{\mathrm{SRS}}}}{\operatorname{MSE}_{\widehat{\alpha}_{\mathrm{RSS}}}} \\
\operatorname{Eff}_{\hat{\theta}}\left(\widehat{\theta}_{\mathrm{RSS}}, \widehat{\theta}_{\mathrm{SRS}}\right) & =\frac{\operatorname{MSE}_{\widehat{\theta}_{\mathrm{SRS}}}}{\operatorname{MSE}_{\widehat{\theta}_{\mathrm{RSS}}}}
\end{aligned}
$$

The results are reported in Tables 1-8 for the parameter estimates (Es), the MSE, and the Eff.

Based on simulation results presented in Tables 1-8, one can observe that

(i) The values of the efficiency given in the tables are larger than 1. Hence, we can say that the suggested RSS estimators perform better than their SRS counterparts based on all methods considered in this study.

(ii) Under the RSS design, where the number of cycles is increasing, the MSE values are decreasing. For example, when $k=5$, the MSEs of the estimators of $\theta$ using the MLE method are 0.015, 0.011, and 0.009 for $n=3,4$, and 5 , respectively.

(iii) Under the RSS design, the MSE is decreasing when the set size $k$ is increasing. For illustration, when $n=5$, the MSEs of estimators of $\alpha$ using AD method are $1.148,0.189$, and 0.082 for $k=5,10$, and 15 , respectively.

(iv) The MSE of the SRS estimators is decreasing when $N=n k$ is increasing.

(v) In most cases, the efficiency is increasing when $k$ is increasing. For instance, from Table 6 , the values of efficiency of $\alpha$ using the CV method when $n=3$ are 2.707, 5.114, and 8.812 for $k=5,10$, and 15 , respectively.

(vi) The MLE estimators perform better than the other methods under RSS and SRS designs for most cases presented in the tables.

(vii) The values of the estimated parameters indicate that the bias values are negligible and go to zero as the sampling size increases.

\section{Application to Real Datasets}

In this section, we illustrate the performance of the suggested estimators based on RSS design for two well-known real datasets. The first dataset presents the survival times (in days) of 72 guinea pigs infected with virulent tubercle bacilli, observed and reported by Bjerkedal [43]. The data were previously studied by Afify et al. [44]. The data observations are

Dataset 1. 0.1, 0.33, 0.44, 0.56, 0.59, 0.72, 0.74, 0.77, 0.92, 0.93, 0.96, 1, 1, 1.02, 1.05, 1.07, 07, .08, 1.08, 1.08, $1.09,1.12,1.13,1.15,1.16,1.2,1.21,1.22,1.22,1.24,1.3$, $1.34,1.36,1.39,1.44,1.46,1.53,1.59,1.6,1.63,1.63$, $1.68,1.71,1.72,1.76,1.83,1.95,1.96,1.97,2.02,2.13$, 2.15, 2.16, 2.22, 2.3, 2.31, 2.4, 2.45, 2.51, 2.53, 2.54, 2.54, $2.78,2.93,3.27,3.42,3.47,3.61,4.02,4.32,4.58,5.55$.

The second dataset represents the number of million revolutions before failure for each of the 23 ball bearings in the life tests. It was presented by Lawless [45], and the data observations are

Dataset 2. 17.88, 28.92, 33.0, 41.52, 42.12, 45.6, 48.8, $51.84,51.96,54.12,55.56,67.8,68.44,68.88,84.12$, $93.12,98.64,105.12,105.84,105.84,127.92,128.04$, 173.4 .

First, we fitted the GQLD model to both datasets. Then, we considered the Kolmogorov-Smirnov (KS) test with its $p$ value for quantifying the distance between the empirical distribution function of the real data and the cumulative distribution function using the estimators' parameters in each dataset. The results are summarized in Table 9. As 
TABLE 1: Es, MSE, and Eff values using the MLE, MPS, and OLS methods for the GQLD parameters with $\alpha=1, \theta=1$ under SRS and RSS.

\begin{tabular}{|c|c|c|c|c|c|c|c|c|c|c|c|c|}
\hline \multirow{2}{*}{$n$} & \multirow{2}{*}{$k$} & & \multirow{2}{*}{ Method } & \multicolumn{3}{|c|}{ MLE } & \multicolumn{3}{|c|}{ MPS } & \multicolumn{3}{|c|}{ OLS } \\
\hline & & & & Es & MSE & Eff & Es & MSE & Eff & Es & MSE & Eff \\
\hline \multirow{12}{*}{3} & \multirow{4}{*}{5} & \multirow{2}{*}{$\widehat{\alpha}$} & SRS & 1.548 & 2.94 & \multirow{2}{*}{2.715} & 1.551 & 3.393 & \multirow{2}{*}{2.307} & 1.581 & 3.099 & \multirow{2}{*}{2.499} \\
\hline & & & RSS & 1.261 & 1.083 & & 1.242 & 1.471 & & 1.296 & 1.240 & \\
\hline & & \multirow{2}{*}{$\hat{\theta}$} & SRS & 1.032 & 0.032 & \multirow{2}{*}{2.133} & 1.068 & 0.039 & \multirow{2}{*}{1.950} & 1.020 & 0.038 & \multirow{2}{*}{2.235} \\
\hline & & & RSS & 1.014 & 0.015 & & 1.054 & 0.020 & & 1.005 & 0.017 & \\
\hline & \multirow{4}{*}{10} & & SRS & 1.288 & 1.243 & \multirow{2}{*}{10.106} & 1.274 & 1.392 & \multirow{2}{*}{9.943} & 1.327 & 1.393 & \multirow{2}{*}{9.476} \\
\hline & & $\alpha$ & RSS & 1.057 & 0.123 & & 1.020 & 0.140 & & 1.072 & 0.147 & \\
\hline & & \multirow{2}{*}{$\widehat{\theta}$} & SRS & 1.017 & 0.013 & \multirow{2}{*}{3.250} & 1.041 & 0.015 & \multirow{2}{*}{3.000} & 1.014 & 0.017 & \\
\hline & & & RSS & 1.005 & 0.004 & & 1.029 & 0.005 & & 1.001 & 0.004 & 4.250 \\
\hline & & & SRS & 1.189 & 0.645 & & 1.168 & 0.681 & & 1.211 & 0.720 & \\
\hline & 15 & $\alpha$ & RSS & 1.024 & 0.048 & 13.438 & 1.000 & 0.062 & 10.984 & 1.033 & 0.053 & 13.585 \\
\hline & 15 & $\widehat{\theta}$ & SRS & 1.013 & 0.010 & 5000 & 1.029 & 0.011 & 5.500 & 1.010 & 0.012 & 6000 \\
\hline & & $\theta$ & RSS & 1.002 & 0.002 & 5.000 & 1.020 & 0.002 & 5.500 & 1.000 & 0.002 & 6.000 \\
\hline & & $\widehat{\alpha}$ & SRS & 1.420 & 2.113 & 2919 & 1.400 & 2.327 & 2577 & 1.439 & 2.224 & 2801 \\
\hline & & & RSS & 1.171 & 0.724 & 2.919 & 1.124 & 0.903 & 2.571 & 1.204 & 0.794 & 2.001 \\
\hline & 5 & $\widehat{\theta}$ & SRS & 1.022 & 0.021 & 1909 & 1.051 & 0.025 & 1786 & 1.014 & 0.026 & \\
\hline & & & RSS & 1.008 & 0.011 & 1.909 & 1.041 & 0.014 & $1 . / 86$ & 1.003 & 0.013 & 2.000 \\
\hline & & $\widehat{\alpha}$ & SRS & 1.195 & 0.687 & 7387 & 1.175 & 0.757 & & 1.216 & 0.739 & \\
\hline 4 & 10 & $\alpha$ & RSS & 1.043 & 0.093 & 7.387 & 1.013 & 0.112 & 6.759 & 1.049 & 0.099 & 7.465 \\
\hline 4 & 10 & $\widehat{\theta}$ & SRS & 1.017 & 0.010 & & 1.035 & 0.011 & & 1.011 & 0.012 & \\
\hline & & $\theta$ & RSS & 1.003 & 0.003 & 3.333 & 1.023 & 0.004 & 2.750 & 1.000 & 0.003 & 4.000 \\
\hline & & $\widehat{\alpha}$ & SRS & 1.098 & 0.328 & 7810 & 1.079 & 0.347 & 7229 & 1.103 & 0.339 & 7370 \\
\hline & 15 & $\alpha$ & RSS & 1.014 & 0.042 & 7.810 & 0.991 & 0.048 & 7.229 & 1.017 & 0.046 & 7.370 \\
\hline & 15 & $\widehat{\theta}$ & SRS & 1.004 & 0.007 & 7000 & 1.018 & 0.007 & 3500 & 1.001 & 0.008 & 4000 \\
\hline & & & RSS & 1.000 & 0.001 & 1.000 & 1.015 & 0.002 & 3.500 & 0.998 & 0.002 & 4.000 \\
\hline & & & SRS & 1.376 & 1.845 & 4020 & 1.364 & 2.026 & 3033 & 1.393 & 1.851 & 3553 \\
\hline & 5 & $\alpha$ & RSS & 1.128 & 0.459 & 4.020 & 1.111 & 0.668 & 3.033 & 1.155 & 0.521 & 3.553 \\
\hline & 5 & $\widehat{\theta}$ & SRS & 1.018 & 0.020 & 2020 & 1.044 & 0.022 & م000 & 1.013 & 0.023 & 2300 \\
\hline & & & RSS & 1.005 & 0.009 & 2.222 & 1.033 & 0.011 & 2.000 & 1.001 & 0.010 & 2.300 \\
\hline & & & SRS & 1.158 & 0.555 & & 1.138 & 0.595 & & 1.162 & 0.539 & \\
\hline 5 & & $\bar{\alpha}$ & RSS & 1.036 & 0.082 & 6.768 & 1.012 & 0.096 & 6.198 & 1.044 & 0.091 & 5.923 \\
\hline 5 & 10 & $\widehat{\theta}$ & SRS & 1.009 & 0.009 & & 1.024 & 0.010 & & 1.005 & 0.010 & 3333 \\
\hline & & $\theta$ & RSS & 1.003 & 0.002 & 4.500 & 1.021 & 0.003 & 3.333 & 1.001 & 0.003 & 3.333 \\
\hline & & & SRS & 1.073 & 0.241 & & 1.056 & 0.248 & & 1.075 & 0.245 & \\
\hline & & $\bar{\alpha}$ & RSS & 1.017 & 0.031 & 7.774 & 0.998 & 0.037 & 6.703 & 1.021 & 0.034 & 7.206 \\
\hline & 15 & $\widehat{\theta}$ & SRS & 1.004 & 0.006 & & 1.015 & 0.006 & & 1.000 & 0.007 & \\
\hline & & $\theta$ & RSS & 1.002 & 0.001 & 6.000 & 1.015 & 0.002 & 3.000 & 1.000 & 0.001 & 7.000 \\
\hline
\end{tabular}

shown in this table, the $p$ value for the corresponding critical value in each dataset is greater than $5 \%$, which indicates that the GQLD model fitted both datasets well.

To show the superiority of RSS over the SRS using the different estimation methods, we considered the Kolmogorov-Smirnov (KS) test but now for quantifying the distance between the empirical distribution function of the real data and the cumulative distribution function using the estimators' parameters in each design, based on the choice of $n$ and $k$. Note that we used the KS here as an alternative to the mean squared error and relative bias, and it is defined in our case as

$$
\mathrm{KS}=\max \left\{\max _{1 \leq i \leq n}\left[\frac{i}{n}-F\left(x_{(i)}, \widehat{\theta}, \widehat{\alpha}\right)\right], \max _{1 \leq i \leq n}\left[F\left(x_{(i)}, \widehat{\theta}, \widehat{\alpha}\right)-\frac{i-1}{n}\right]\right\},
$$

where $n$ is the sample size. Of course, estimators with lower KS values and higher $p$ value (greater than $5 \%$ ) are better than the other competitions. Recall that the MLE estimators based on SRS for all datasets are considered the real population parameters.
For the first dataset, we considered the SRS design, and for each estimation method, we calculate the estimators using a sample of size $n k=12$. Then, we used a sample of sizes $n=2$ and $k=6$ for calculating estimators using the RSS design, and based on the cycles shown in Table 10, we compare SRS and RSS designs in terms of the KS distance value and $p$ value. The results are given in Table 11, and the corresponding fittings are displayed in Figure 1.

For the second dataset, the sample size is selected as $n k=$ 8 in the SRS design, while we used $n=2$ and $k=4$ for calculating estimators based on the RSS design using the cycles in Table 12. The estimators' values, KS distance, and $p$ values are computed and summarized in Table 13, and the corresponding fittings are displayed in Figure 2.

The results in Tables 11 and 13 indicate that for the estimates based on the RSS design, the KS distance values are less than their counterparts using SRS design, and the corresponding $p$ values based on the RSS estimators are greater than those by SRS design. Figures 1 and 2 support this claim. 
TABLE 2: Es, MSE, and Eff values using the WLS, CV, and AD methods for the GQLD model parameters with $\alpha=1, \theta=1$ under SRS and RSS.

\begin{tabular}{|c|c|c|c|c|c|c|c|c|c|c|c|c|}
\hline \multirow{2}{*}{$n$} & \multirow{2}{*}{$k$} & & \multirow{2}{*}{ Method } & \multicolumn{3}{|c|}{ WLS } & \multicolumn{3}{|c|}{$\mathrm{CV}$} & \multicolumn{3}{|c|}{$\mathrm{AD}$} \\
\hline & & & & Es & MSE & Eff & Es & MSE & Eff & Es & MSE & Eff \\
\hline \multirow{12}{*}{3} & \multirow{4}{*}{5} & \multirow{2}{*}{$\widehat{\alpha}$} & SRS & 1.564 & 2.945 & \multirow{2}{*}{2.694} & 1.556 & 2.982 & \multirow{2}{*}{2.696} & 1.555 & 2.863 & \multirow{2}{*}{2.706} \\
\hline & & & RSS & 1.274 & 1.093 & & 1.260 & 1.106 & & 1.274 & 1.058 & \\
\hline & & \multirow{2}{*}{$\widehat{\theta}$} & SRS & 1.019 & 0.036 & \multirow{2}{*}{2.250} & 1.027 & 0.037 & \multirow{2}{*}{2.312} & 1.019 & 0.033 & \multirow{2}{*}{2.200} \\
\hline & & & RSS & 1.003 & 0.016 & & 1.012 & 0.016 & & 1.005 & 0.015 & \\
\hline & \multirow{4}{*}{10} & \multirow{2}{*}{$\widehat{\alpha}$} & SRS & 1.309 & 1.296 & \multirow{2}{*}{9.818} & 1.302 & 1.310 & \multirow{2}{*}{10.000} & 1.305 & 1.252 & \multirow{2}{*}{10.097} \\
\hline & & & RSS & 1.068 & 0.132 & & 1.058 & 0.131 & & 1.063 & 0.124 & \\
\hline & & \multirow{2}{*}{$\widehat{\theta}$} & SRS & 1.013 & 0.016 & \multirow{2}{*}{4.000} & 1.018 & 0.016 & & 1.013 & 0.015 & \\
\hline & & & RSS & 1.000 & 0.004 & & 1.005 & 0.004 & 4.000 & 1.001 & 0.004 & 3.750 \\
\hline & & $\widehat{\alpha}$ & SRS & 1.197 & 0.635 & & 1.191 & 0.638 & & 1.197 & 0.637 & \\
\hline & 15 & $\alpha$ & RSS & 1.030 & 0.050 & 12.700 & 1.023 & 0.050 & 12.760 & 1.028 & 0.048 & 13.271 \\
\hline & 15 & $\widehat{A}$ & SRS & 1.010 & 0.011 & & 1.013 & 0.011 & & 1.009 & 0.011 & \\
\hline & & & RSS & 0.999 & 0.002 & 5.500 & 1.003 & 0.002 & 5.500 & 1.000 & 0.002 & 5.500 \\
\hline & & $\widehat{\alpha}$ & SRS & 1.431 & 2.163 & 2935 & 1.425 & 2.189 & 2966 & 1.424 & 2.082 & 2900 \\
\hline & 5 & $\alpha$ & RSS & 1.189 & 0.737 & 2.935 & 1.175 & 0.738 & 2.966 & 1.183 & 0.718 & 2.900 \\
\hline & 5 & $\widehat{\theta}$ & SRS & 1.012 & 0.024 & & 1.019 & 0.024 & & 1.013 & 0.022 & \\
\hline & & $\theta$ & RSS & 1.001 & 0.012 & 2.000 & 1.008 & 0.012 & 2.000 & 1.001 & 0.011 & 2.000 \\
\hline & & & SRS & 1.211 & 0.699 & & 1.205 & 0.706 & & 1.205 & 0.677 & \\
\hline & & $\bar{\alpha}$ & RSS & 1.050 & 0.095 & 7.358 & 1.042 & 0.095 & 7.432 & 1.048 & 0.093 & 7.280 \\
\hline 4 & 10 & $\widehat{\theta}$ & SRS & 1.012 & 0.011 & & 1.015 & 0.011 & & 1.011 & 0.01 & \\
\hline & & $\theta$ & RSS & 1.000 & 0.003 & 3.667 & 1.004 & 0.003 & 3.667 & 1.001 & 0.003 & 3.333 \\
\hline & & & SRS & 1.101 & 0.323 & & 1.096 & 0.324 & & 1.100 & 0.318 & \\
\hline & 15 & $\alpha$ & RSS & 1.017 & 0.043 & 7.512 & 1.011 & 0.043 & 7.535 & 1.016 & 0.042 & 7.571 \\
\hline & 15 & $\widehat{\theta}$ & SRS & 1.002 & 0.007 & 7000 & 1.004 & 0.007 & 7000 & 1.002 & 0.007 & \\
\hline & & & RSS & 0.998 & 0.001 & 7.000 & 1.001 & 0.001 & 7.000 & 0.999 & 0.001 & 7.000 \\
\hline & & & SRS & 1.386 & 1.810 & & 1.380 & 1.840 & & 1.381 & 1.792 & \\
\hline & & $\widehat{\alpha}$ & RSS & 1.147 & 0.498 & 3.635 & 1.135 & 0.504 & 3.651 & 1.139 & 0.458 & 3.913 \\
\hline & 5 & $\widehat{\theta}$ & SRS & 1.012 & 0.021 & & 1.018 & 0.022 & & 1.011 & 0.020 & \\
\hline & & $\theta$ & RSS & 1.000 & 0.009 & 2.333 & 1.006 & 0.009 & 2.444 & 1.000 & 0.009 & 2.222 \\
\hline & & & SRS & 1.160 & 0.523 & 6226 & 1.155 & 0.529 & & 1.157 & 0.513 & 5256 \\
\hline & & $\alpha$ & RSS & 1.042 & 0.084 & 6.226 & 1.035 & 0.084 & 6.298 & 1.040 & 0.082 & 6.256 \\
\hline 5 & 10 & $\widehat{\theta}$ & SRS & 1.005 & 0.010 & & 1.008 & 0.010 & & 1.005 & 0.010 & \\
\hline & & $\theta$ & RSS & 1.001 & 0.003 & 3.333 & 1.004 & 0.003 & 3.333 & 1.001 & 0.003 & 3.333 \\
\hline & & & SRS & 1.073 & 0.236 & & 1.069 & 0.237 & & 1.074 & 0.237 & \\
\hline & & $\alpha$ & RSS & 1.021 & 0.031 & 7.613 & 1.016 & 0.031 & 7.645 & 1.019 & 0.031 & 7.645 \\
\hline & 15 & $\widehat{\theta}$ & SRS & 1.000 & 0.006 & & 1.002 & 0.006 & & 1.000 & 0.006 & \\
\hline & & $\theta$ & RSS & 1.000 & 0.001 & 6.000 & 1.003 & 0.001 & 6.000 & 1.001 & 0.001 & 6.000 \\
\hline
\end{tabular}

TABLE 3: Es, MSE, and Eff values using the MLE, MPS, and OLS methods for the GQLD model parameters with $\alpha=1, \theta=3$ under SRS and RSS.

\begin{tabular}{|c|c|c|c|c|c|c|c|c|c|c|c|c|}
\hline \multirow{2}{*}{$n$} & \multirow{2}{*}{$k$} & & \multirow{2}{*}{ Method } & \multicolumn{3}{|c|}{ MLE } & \multicolumn{3}{|c|}{ MPS } & \multicolumn{3}{|c|}{ OLS } \\
\hline & & & & Es & MSE & Eff & Es & MSE & Eff & Es & MSE & Eff \\
\hline \multirow{12}{*}{3} & \multirow{4}{*}{5} & \multirow{2}{*}{$\widehat{\alpha}$} & SRS & 1.523 & 2.913 & \multirow{2}{*}{2.715} & 1.533 & 3.385 & \multirow{2}{*}{2.287} & 1.591 & 3.074 & \multirow{2}{*}{2.465} \\
\hline & & & RSS & 1.268 & 1.073 & & 1.249 & 1.480 & & 1.312 & 1.247 & \\
\hline & & \multirow{2}{*}{$\hat{\theta}$} & SRS & 3.082 & 0.279 & \multirow{2}{*}{2.098} & 3.192 & 0.337 & \multirow{2}{*}{1.872} & 3.062 & 0.339 & \multirow{2}{*}{2.159} \\
\hline & & & RSS & 3.048 & 0.133 & & 3.164 & 0.180 & & 3.025 & 0.157 & \\
\hline & \multirow{4}{*}{10} & \multirow{2}{*}{$\widehat{\alpha}$} & SRS & 1.235 & 1.059 & \multirow{2}{*}{8.209} & 1.210 & 1.142 & \multirow{2}{*}{7.513} & 1.243 & 1.069 & \multirow{2}{*}{7.476} \\
\hline & & & RSS & 1.055 & 0.129 & & 1.012 & 0.152 & & 1.068 & 0.143 & \\
\hline & & $\widehat{\theta}$ & SRS & 3.041 & 0.123 & \multirow[b]{2}{*}{3.514} & 3.106 & 0.141 & \multirow{2}{*}{3.000} & 3.015 & 0.145 & \multirow{2}{*}{3.816} \\
\hline & & $\theta$ & RSS & 3.012 & 0.035 & & 3.082 & 0.047 & & 3.003 & 0.038 & \\
\hline & \multirow{4}{*}{15} & $\widehat{\alpha}$ & SRS & 1.191 & 0.63 & \multirow{2}{*}{13.125} & 1.169 & 0.672 & \multirow{2}{*}{11.200} & 1.212 & 0.709 & \multirow{2}{*}{13.130} \\
\hline & & $a$ & RSS & 1.027 & 0.048 & & 1.001 & 0.060 & & 1.033 & 0.054 & \\
\hline & & \multirow{2}{*}{$\widehat{\theta}$} & SRS & 3.044 & 0.084 & \multirow{2}{*}{6.000} & 3.094 & 0.094 & \multirow{2}{*}{4.273} & 3.034 & 0.102 & \multirow{2}{*}{6.375} \\
\hline & & & RSS & 3.007 & 0.014 & & 3.064 & 0.022 & & 3.000 & 0.016 & \\
\hline
\end{tabular}


TABle 3: Continued.

\begin{tabular}{|c|c|c|c|c|c|c|c|c|c|c|c|c|}
\hline \multirow{2}{*}{$n$} & \multirow{2}{*}{$k$} & & \multirow{2}{*}{ Method } & \multicolumn{3}{|c|}{ MLE } & \multicolumn{3}{|c|}{ MPS } & \multicolumn{3}{|c|}{ OLS } \\
\hline & & & & Es & MSE & Eff & Es & MSE & Eff & Es & MSE & Eff \\
\hline \multirow{12}{*}{4} & \multirow{4}{*}{5} & \multirow{2}{*}{$\widehat{\alpha}$} & SRS & 1.402 & 2.025 & \multirow{2}{*}{4.175} & 1.397 & 2.339 & \multirow[t]{2}{*}{3.566} & 1.431 & 2.132 & \multirow[t]{2}{*}{3.891} \\
\hline & & & RSS & 1.094 & 0.485 & & 1.042 & 0.656 & & 1.126 & 0.548 & \\
\hline & & \multirow{2}{*}{$\widehat{\theta}$} & SRS & 3.064 & 0.192 & \multirow{2}{*}{2.286} & 3.153 & 0.228 & \multirow{2}{*}{2.131} & 3.039 & 0.243 & \multirow{2}{*}{2.455} \\
\hline & & & RSS & 2.998 & 0.084 & & 3.092 & 0.107 & & 2.981 & 0.099 & \\
\hline & \multirow{4}{*}{10} & \multirow{2}{*}{$\widehat{\alpha}$} & SRS & 1.143 & 0.579 & \multirow{2}{*}{6.095} & 1.121 & 0.645 & \multirow{2}{*}{5.513} & 1.163 & 0.637 & \multirow{2}{*}{6.307} \\
\hline & & & RSS & 1.045 & 0.095 & & 1.011 & 0.117 & & 1.052 & 0.101 & \\
\hline & & \multirow{2}{*}{$\widehat{\theta}$} & SRS & 3.029 & 0.087 & \multirow{2}{*}{3.346} & 3.082 & 0.097 & \multirow{2}{*}{2.553} & 3.008 & 0.103 & \multirow{2}{*}{3.679} \\
\hline & & & RSS & 3.012 & 0.026 & & 3.073 & 0.038 & & 3.002 & 0.028 & \\
\hline & \multirow{4}{*}{15} & $\widehat{\hat{\alpha}}$ & SRS & 1.106 & 0.333 & \multirow{2}{*}{9.000} & 1.087 & 0.353 & \multirow{2}{*}{8.209} & 1.117 & 0.366 & \multirow{2}{*}{8.927} \\
\hline & & $\alpha$ & RSS & 1.009 & 0.037 & & 0.985 & 0.043 & & 1.013 & 0.041 & \\
\hline & & $\widehat{\theta}$ & SRS & 3.022 & 0.058 & & 3.063 & 0.064 & & 3.011 & 0.070 & \\
\hline & & $\theta$ & RSS & 3.000 & 0.011 & 5.273 & 3.042 & 0.016 & 4.000 & 2.994 & 0.013 & 5.385 \\
\hline & & & SRS & 1.330 & 1.321 & & 1.314 & 1.480 & & 1.362 & 1.417 & \\
\hline & 5 & $\widehat{\alpha}$ & RSS & 1.118 & 0.401 & 3.294 & 1.095 & 0.519 & 2.852 & 1.141 & 0.440 & 3.220 \\
\hline & 5 & $\widehat{\theta}$ & SRS & 3.066 & 0.159 & & 3.145 & 0.186 & & 3.051 & 0.182 & \\
\hline & & $\theta$ & RSS & 3.025 & 0.072 & 2.208 & 3.116 & 0.099 & 1.879 & 3.010 & 0.082 & 2.220 \\
\hline & & & SRS & 1.110 & 0.400 & & 1.087 & 0.421 & & 1.122 & 0.420 & \\
\hline & & $\widehat{\alpha}$ & RSS & 1.017 & 0.066 & 6.061 & 0.991 & 0.079 & 5.329 & 1.023 & 0.070 & 6.000 \\
\hline 5 & 10 & $\widehat{A}$ & SRS & 3.021 & 0.070 & & 3.067 & 0.077 & & 3.006 & 0.083 & \\
\hline & & $\theta$ & RSS & 3.001 & 0.019 & 3.684 & 3.051 & 0.025 & 3.080 & 2.993 & 0.021 & 3.952 \\
\hline & & & SRS & 1.098 & 0.230 & & 1.081 & 0.238 & & 1.115 & 0.272 & \\
\hline & & $\bar{\alpha}$ & RSS & 1.019 & 0.031 & 7.419 & 0.996 & 0.036 & 6.611 & 1.023 & 0.034 & 8.000 \\
\hline & 15 & $\widehat{\theta}$ & SRS & 3.019 & 0.047 & & 3.054 & 0.051 & & 3.020 & 0.059 & \\
\hline & & $\theta$ & RSS & 3.007 & 0.009 & 5.222 & 3.042 & 0.013 & 3.923 & 3.002 & 0.010 & 5.900 \\
\hline
\end{tabular}

TABLe 4: Es, MSE, and Eff values using the WLS, CV, and AD methods for the GQLD model parameters with $\alpha=1, \theta=3$ under SRS and RSS.

\begin{tabular}{|c|c|c|c|c|c|c|c|c|c|c|c|c|}
\hline \multirow{2}{*}{$n$} & \multirow{2}{*}{$k$} & & \multirow{2}{*}{ Method } & \multicolumn{3}{|c|}{ WLS } & \multicolumn{3}{|c|}{$\mathrm{CV}$} & \multicolumn{3}{|c|}{$\mathrm{AD}$} \\
\hline & & & & Es & MSE & Eff & Es & MSE & Eff & Es & MSE & Eff \\
\hline \multirow{12}{*}{3} & \multirow{4}{*}{5} & \multirow{2}{*}{$\widehat{\alpha}$} & SRS & 1.568 & 2.927 & \multirow{2}{*}{2.468} & 1.556 & 2.957 & \multirow{2}{*}{2.436} & 1.544 & 2.855 & \multirow{2}{*}{2.704} \\
\hline & & & RSS & 1.301 & 1.186 & & 1.290 & 1.214 & & 1.283 & 1.056 & \\
\hline & & \multirow{2}{*}{$\widehat{\theta}$} & SRS & 3.054 & 0.317 & \multirow{2}{*}{2.142} & 3.079 & 0.321 & \multirow{2}{*}{2.140} & 3.049 & 0.296 & \multirow{2}{*}{2.193} \\
\hline & & & RSS & 3.020 & 0.148 & & 3.046 & 0.150 & & 3.019 & 0.135 & \\
\hline & \multirow{4}{*}{10} & $\widehat{\alpha}$ & SRS & 1.239 & 1.031 & \multirow{2}{*}{7.931} & 1.234 & 1.049 & \multirow{2}{*}{8.069} & 1.238 & 1.026 & \multirow{2}{*}{7.892} \\
\hline & & $\alpha$ & RSS & 1.064 & 0.130 & & 1.054 & 0.130 & & 1.061 & 0.130 & \\
\hline & & \multirow{2}{*}{$\widehat{\theta}$} & SRS & 3.016 & 0.136 & \multirow{2}{*}{3.886} & 3.031 & 0.137 & \multirow{2}{*}{3.806} & 3.017 & 0.132 & \multirow{2}{*}{3.771} \\
\hline & & & RSS & 3.000 & 0.035 & & 3.015 & 0.036 & & 3.001 & 0.035 & \\
\hline & \multirow{4}{*}{15} & $\widehat{\alpha}$ & SRS & 1.203 & 0.646 & \multirow{2}{*}{12.667} & 1.197 & 0.650 & \multirow{2}{*}{13.000} & 1.199 & 0.634 & \multirow{2}{*}{12.939} \\
\hline & & $\alpha$ & RSS & 1.033 & 0.051 & & 1.026 & 0.050 & & 1.030 & 0.049 & \\
\hline & & \multirow{2}{*}{$\hat{\theta}$} & SRS & 3.033 & 0.095 & & 3.044 & 0.096 & & 3.031 & 0.092 & \\
\hline & & & RSS & 2.999 & 0.015 & 6.333 & 3.010 & 0.015 & 6.400 & 3.001 & 0.014 & 6.571 \\
\hline & & & SRS & 1.416 & 2.001 & 3863 & 1.409 & 2.034 & 3919 & 1.407 & 1.950 & 4088 \\
\hline & & $\alpha$ & RSS & 1.115 & 0.518 & 3.863 & 1.101 & 0.519 & 3.919 & 1.106 & 0.477 & 4.088 \\
\hline & 5 & $\widehat{\theta}$ & SRS & 3.036 & 0.229 & & 3.056 & 0.230 & & 3.035 & 0.208 & \\
\hline & & $\theta$ & RSS & 2.976 & 0.092 & 2.489 & 2.997 & 0.092 & 2.500 & 2.976 & 0.086 & 2.419 \\
\hline & & & SRS & 1.154 & 0.578 & & 1.147 & 0.584 & & 1.154 & 0.587 & \\
\hline & & $\widehat{\alpha}$ & RSS & 1.053 & 0.097 & 5.959 & 1.045 & 0.097 & 6.021 & 1.050 & 0.096 & 6.115 \\
\hline 4 & 10 & $\widehat{\theta}$ & SRS & 3.009 & 0.095 & & 3.021 & 0.096 & & 3.009 & 0.093 & 3577 \\
\hline & & $\theta$ & RSS & 3.003 & 0.027 & 3.519 & 3.015 & 0.027 & 3.556 & 3.004 & 0.026 & 3.577 \\
\hline & & & SRS & 1.111 & 0.338 & & 1.106 & 0.338 & & 1.111 & 0.333 & \\
\hline & & $\bar{\alpha}$ & RSS & 1.013 & 0.038 & 8.895 & 1.007 & 0.038 & 8.895 & 1.012 & 0.037 & 9.000 \\
\hline & 15 & $\widehat{\theta}$ & SRS & 3.012 & 0.065 & & 3.020 & 0.065 & & 3.011 & 0.064 & \\
\hline & & $\theta$ & RSS & 2.993 & 0.012 & 5.417 & 3.002 & 0.012 & 5.417 & 2.995 & 0.012 & 5.333 \\
\hline
\end{tabular}


TABle 4: Continued.

\begin{tabular}{|c|c|c|c|c|c|c|c|c|c|c|c|c|}
\hline \multirow{2}{*}{$n$} & \multirow{2}{*}{$k$} & & \multirow{2}{*}{ Method } & \multicolumn{3}{|c|}{ WLS } & \multicolumn{3}{|c|}{$\mathrm{CV}$} & \multicolumn{3}{|c|}{$\mathrm{AD}$} \\
\hline & & & & Es & MSE & Eff & Es & MSE & Eff & Es & MSE & Eff \\
\hline \multirow{12}{*}{5} & \multirow{4}{*}{5} & \multirow{2}{*}{$\widehat{\alpha}$} & SRS & 1.347 & 1.291 & \multirow{2}{*}{3.126} & 1.338 & 1.299 & \multirow{2}{*}{3.138} & 1.338 & 1.276 & \multirow{2}{*}{3.198} \\
\hline & & & RSS & 1.134 & 0.413 & & 1.123 & 0.414 & & 1.128 & 0.399 & \\
\hline & & \multirow{2}{*}{$\hat{\theta}$} & SRS & 3.048 & 0.172 & \multirow{2}{*}{2.263} & 3.065 & 0.174 & \multirow{2}{*}{2.260} & 3.047 & 0.164 & \multirow{2}{*}{2.247} \\
\hline & & & RSS & 3.008 & 0.076 & & 3.025 & 0.077 & & 3.007 & 0.073 & \\
\hline & \multirow{4}{*}{10} & \multirow{2}{*}{$\widehat{\alpha}$} & SRS & 1.119 & 0.400 & \multirow{2}{*}{5.970} & 1.114 & 0.404 & \multirow{2}{*}{6.030} & 1.114 & 0.385 & \multirow{2}{*}{5.833} \\
\hline & & & RSS & 1.023 & 0.067 & & 1.016 & 0.067 & & 1.022 & 0.066 & \\
\hline & & $\widehat{\theta}$ & SRS & 3.007 & 0.077 & \multirow{2}{*}{3.850} & 3.017 & 0.078 & \multirow{2}{*}{3.900} & 3.005 & 0.076 & \multirow{2}{*}{4.000} \\
\hline & & $\theta$ & RSS & 2.993 & 0.020 & & 3.003 & 0.020 & & 2.994 & 0.019 & \\
\hline & \multirow{4}{*}{15} & & SRS & 1.107 & 0.247 & \multirow{2}{*}{7.719} & 1.103 & 0.246 & \multirow{2}{*}{7.688} & 1.106 & 0.244 & \multirow{2}{*}{7.871} \\
\hline & & $\alpha$ & RSS & 1.022 & 0.032 & & 1.017 & 0.032 & & 1.021 & 0.031 & \\
\hline & & \multirow{2}{*}{$\widehat{\theta}$} & SRS & 3.018 & 0.054 & \multirow{2}{*}{5.400} & 3.025 & 0.054 & \multirow{2}{*}{5.400} & 3.016 & 0.053 & \multirow{2}{*}{5.889} \\
\hline & & & RSS & 3.002 & 0.010 & & 3.009 & 0.010 & & 3.003 & 0.009 & \\
\hline
\end{tabular}

TABLE 5: Es, MSE, and Eff values using the MLE, MPS, and OLS methods for the GQLD model parameters with $\alpha=0.5, \theta=1$ under SRS and RSS.

\begin{tabular}{|c|c|c|c|c|c|c|c|c|c|c|c|c|}
\hline \multirow{2}{*}{$n$} & \multirow{2}{*}{$k$} & & \multirow{2}{*}{ Method } & \multicolumn{3}{|c|}{ MLE } & \multicolumn{3}{|c|}{ MPS } & \multicolumn{3}{|c|}{ OLS } \\
\hline & & & & Es & MSE & Eff & Es & MSE & Eff & Es & MSE & Eff \\
\hline \multirow{12}{*}{3} & \multirow{4}{*}{5} & & SRS & 0.702 & 0.727 & \multirow{2}{*}{2.713} & 0.658 & 0.806 & \multirow{2}{*}{2.858} & 0.759 & 0.857 & \multirow{2}{*}{2.738} \\
\hline & & $\alpha$ & RSS & 0.610 & 0.268 & & 0.534 & 0.282 & & 0.638 & 0.313 & \\
\hline & & \multirow{2}{*}{$\widehat{\theta}$} & SRS & 1.021 & 0.024 & \multirow{2}{*}{1.846} & 1.053 & 0.028 & \multirow{2}{*}{1.647} & 1.013 & 0.030 & \multirow{2}{*}{2.000} \\
\hline & & & RSS & 1.015 & 0.013 & & 1.049 & 0.017 & & 1.006 & 0.015 & \\
\hline & \multirow{4}{*}{10} & & SRS & 0.585 & 0.213 & \multirow{2}{*}{5.071} & 0.543 & 0.219 & \multirow{2}{*}{4.380} & 0.607 & 0.249 & \multirow{2}{*}{5.082} \\
\hline & & $\alpha$ & RSS & 0.520 & 0.042 & & 0.470 & 0.050 & & 0.531 & 0.049 & \\
\hline & & \multirow{2}{*}{$\widehat{\theta}$} & SRS & 1.013 & 0.012 & \multirow{2}{*}{3.000} & 1.033 & 0.014 & \multirow{2}{*}{2.800} & 1.005 & 0.014 & \multirow{2}{*}{3.500} \\
\hline & & & RSS & 1.003 & 0.004 & & 1.025 & 0.005 & & 0.999 & 0.004 & \\
\hline & & & SRS & 0.574 & 0.132 & & 0.539 & 0.131 & & 0.590 & 0.152 & \\
\hline & 15 & $\alpha$ & RSS & 0.513 & 0.015 & 8.800 & 0.474 & 0.019 & 6.895 & 0.518 & 0.017 & 8.941 \\
\hline & 15 & $\widehat{\theta}$ & SRS & 1.014 & 0.008 & & 1.029 & 0.009 & & 1.011 & 0.010 & \\
\hline & & $\theta$ & RSS & 1.003 & 0.001 & 8.000 & 1.019 & 0.002 & 4.500 & 1.000 & 0.002 & 5.000 \\
\hline & & & SRS & 0.649 & 0.507 & & 0.607 & 0.558 & & 0.677 & 0.503 & \\
\hline & & $\alpha$ & RSS & 0.540 & 0.113 & 4.487 & 0.466 & 0.124 & 4.500 & 0.558 & 0.122 & 4.123 \\
\hline & 5 & $\widehat{\theta}$ & SRS & 1.015 & 0.018 & 2250 & 1.042 & 0.021 & & 1.007 & 0.022 & \\
\hline & & & RSS & 1.002 & 0.008 & 2.250 & 1.029 & 0.010 & 2.100 & 0.996 & 0.009 & 2.444 \\
\hline & & & SRS & 0.551 & 0.121 & & 0.514 & 0.120 & & 0.570 & 0.140 & \\
\hline & & $\widehat{\alpha}$ & RSS & 0.520 & 0.028 & 4.321 & 0.477 & 0.033 & 3.636 & 0.530 & 0.033 & 4.242 \\
\hline 4 & 10 & $\widehat{\theta}$ & SRS & 1.008 & 0.008 & & 1.024 & 0.009 & & 1.004 & 0.010 & \\
\hline & & & RSS & 1.004 & 0.002 & 4.000 & 1.022 & 0.003 & 3.000 & 1.002 & 0.003 & 3.333 \\
\hline & & & SRS & 0.535 & 0.079 & & 0.508 & 0.079 & & 0.551 & 0.093 & \\
\hline & & $\alpha$ & RSS & 0.504 & 0.012 & 6.583 & 0.471 & 0.015 & 5.267 & 0.508 & 0.013 & 7.154 \\
\hline & 15 & $\widehat{\theta}$ & SRS & 1.006 & 0.006 & & 1.018 & 0.006 & & 1.003 & 0.007 & \\
\hline & & $\theta$ & RSS & 1.000 & 0.001 & 6.000 & 1.013 & 0.001 & 6.000 & 0.999 & 0.001 & 7.000 \\
\hline & & $\widehat{\alpha}$ & SRS & 0.650 & 0.378 & 4447 & 0.606 & 0.392 & 3920 & 0.683 & 0.424 & 4283 \\
\hline & & $\alpha$ & RSS & 0.542 & 0.085 & 4.447 & 0.491 & 0.100 & 3.920 & 0.563 & 0.099 & 4.283 \\
\hline & 5 & $\widehat{A}$ & SRS & 1.022 & 0.016 & & 1.045 & 0.019 & & 1.018 & 0.018 & \\
\hline & & $\theta$ & RSS & 1.008 & 0.007 & 2.286 & 1.035 & 0.009 & 2.111 & 1.003 & 0.008 & 2.250 \\
\hline & & & SRS & 0.547 & 0.091 & & 0.514 & 0.089 & & 0.561 & 0.102 & \\
\hline & & $\widehat{\alpha}$ & RSS & 0.504 & 0.022 & 4.136 & 0.466 & 0.026 & 3.423 & 0.510 & 0.024 & 4.250 \\
\hline 5 & 10 & $\widehat{\theta}$ & SRS & 1.008 & 0.007 & & 1.022 & 0.008 & & 1.005 & 0.008 & \\
\hline & & $\theta$ & RSS & 1.000 & 0.002 & 3.500 & 1.014 & 0.002 & 4.000 & 0.997 & 0.002 & 4.000 \\
\hline & & & SRS & 0.534 & 0.063 & & 0.509 & 0.063 & & 0.547 & 0.079 & \\
\hline & & $\widehat{\alpha}$ & RSS & 0.506 & 0.010 & 6.300 & 0.479 & 0.013 & 4.846 & 0.509 & 0.011 & 7.182 \\
\hline & 15 & $\widehat{\theta}$ & SRS & 1.004 & 0.004 & & 1.014 & 0.005 & & 1.004 & 0.006 & \\
\hline & & $\theta$ & RSS & 1.001 & 0.001 & 4.000 & 1.012 & 0.001 & 5.000 & 1.000 & 0.001 & 6.000 \\
\hline
\end{tabular}


TABLE 6: Es, MSE, and Eff values using the WLS, CV, and AD methods for the GQLD model parameters with $\alpha=0.5, \theta=1$ under SRS and RSS.

\begin{tabular}{|c|c|c|c|c|c|c|c|c|c|c|c|c|}
\hline \multirow{2}{*}{$n$} & \multirow{2}{*}{$k$} & & \multirow{2}{*}{ Method } & \multicolumn{3}{|c|}{ WLS } & \multicolumn{3}{|c|}{$\mathrm{CV}$} & \multicolumn{3}{|c|}{$\mathrm{AD}$} \\
\hline & & & & Es & MSE & Eff & Es & MSE & Eff & Es & MSE & Eff \\
\hline \multirow{12}{*}{3} & \multirow{4}{*}{5} & \multirow{2}{*}{$\widehat{\alpha}$} & SRS & 0.747 & 0.788 & \multirow{2}{*}{2.680} & 0.730 & 0.777 & \multirow{2}{*}{2.707} & 0.732 & 0.739 & \multirow{2}{*}{2.707} \\
\hline & & & RSS & 0.634 & 0.294 & & 0.617 & 0.287 & & 0.629 & 0.273 & \\
\hline & & \multirow{2}{*}{$\widehat{\theta}$} & SRS & 1.011 & 0.028 & \multirow{2}{*}{2.000} & 1.018 & 0.028 & \multirow{2}{*}{2.000} & 1.010 & 0.026 & \multirow{2}{*}{2.000} \\
\hline & & & RSS & 1.005 & 0.014 & & 1.013 & 0.014 & & 1.006 & 0.013 & \\
\hline & \multirow{4}{*}{10} & \multirow{2}{*}{$\widehat{\alpha}$} & SRS & 0.602 & 0.228 & \multirow{2}{*}{5.067} & 0.592 & 0.225 & \multirow{2}{*}{5.114} & 0.602 & 0.226 & \multirow{2}{*}{5.136} \\
\hline & & & RSS & 0.530 & 0.045 & & 0.519 & 0.044 & & 0.527 & 0.044 & \\
\hline & & \multirow{2}{*}{$\widehat{\theta}$} & SRS & 1.005 & 0.013 & \multirow{2}{*}{3.250} & 1.010 & 0.013 & & 1.006 & 0.013 & \\
\hline & & & RSS & 0.999 & 0.004 & & 1.003 & 0.004 & 3.250 & 0.999 & 0.004 & 3.250 \\
\hline & & $\widehat{\alpha}$ & SRS & 0.586 & 0.142 & & 0.578 & 0.141 & & 0.586 & 0.140 & \\
\hline & 15 & $\alpha$ & RSS & 0.519 & 0.016 & $8.8 / 5$ & 0.511 & 0.016 & 8.812 & 0.518 & 0.015 & 9.333 \\
\hline & 15 & $\widehat{A}$ & SRS & 1.010 & 0.009 & & 1.013 & 0.009 & & 1.010 & 0.009 & \\
\hline & & & RSS & 1.000 & 0.001 & 9.000 & 1.003 & 0.001 & 9.000 & 1.001 & 0.001 & 9.000 \\
\hline & & $\widehat{\alpha}$ & SRS & 0.670 & 0.467 & 4026 & 0.657 & 0.468 & 4105 & 0.665 & 0.452 & \\
\hline & 5 & $\alpha$ & RSS & 0.556 & 0.116 & 4.026 & 0.542 & 0.114 & 4.105 & 0.554 & 0.115 & 3.930 \\
\hline & 5 & $\widehat{\theta}$ & SRS & 1.006 & 0.020 & & 1.012 & 0.021 & & 1.007 & 0.019 & \\
\hline & & $\theta$ & RSS & 0.995 & 0.008 & 2.500 & 1.001 & 0.008 & 2.625 & 0.996 & 0.008 & 2.375 \\
\hline & & & SRS & 0.566 & 0.130 & & 0.557 & 0.128 & & 0.565 & 0.128 & \\
\hline & & $\widehat{\alpha}$ & RSS & 0.528 & 0.029 & 4.483 & 0.519 & 0.029 & 4.414 & 0.527 & 0.029 & 4.414 \\
\hline 4 & 10 & $\widehat{A}$ & SRS & 1.004 & 0.009 & & 1.007 & 0.009 & & 1.003 & 0.009 & \\
\hline & & $\theta$ & RSS & 1.002 & 0.003 & 3.000 & 1.005 & 0.003 & 3.000 & 1.002 & 0.003 & 3.000 \\
\hline & & & SRS & 0.546 & 0.086 & & 0.540 & 0.084 & & 0.547 & 0.085 & \\
\hline & & $\alpha$ & RSS & 0.508 & 0.012 & 7.167 & 0.501 & 0.012 & 7.000 & 0.507 & 0.012 & 7.083 \\
\hline & 15 & $\widehat{A}$ & SRS & 1.003 & 0.006 & 6000 & 1.005 & 0.006 & $600 \Omega$ & 1.003 & 0.006 & 6000 \\
\hline & & & RSS & 0.998 & 0.001 & 6.000 & 1.001 & 0.001 & 6.000 & 0.999 & 0.001 & 6.000 \\
\hline & & & SRS & 0.675 & 0.380 & & 0.662 & 0.375 & & 0.670 & 0.383 & 4.303 \\
\hline & & $\bar{\alpha}$ & RSS & 0.559 & 0.093 & 4.086 & 0.546 & 0.091 & 4.121 & 0.557 & 0.089 & 4.303 \\
\hline & 5 & $\widehat{\theta}$ & SRS & 1.017 & 0.017 & & 1.022 & 0.018 & & 1.016 & 0.017 & \\
\hline & & $\theta$ & RSS & 1.002 & 0.007 & 2.429 & 1.007 & 0.007 & 2.571 & 1.002 & 0.007 & 2.429 \\
\hline & & & SRS & 0.559 & 0.097 & & 0.552 & 0.096 & & 0.557 & 0.096 & \\
\hline & & $\alpha$ & RSS & 0.511 & 0.022 & 4.409 & 0.503 & 0.022 & 4.364 & 0.509 & 0.022 & 4.364 \\
\hline 5 & 10 & $\widehat{\theta}$ & SRS & 1.005 & 0.008 & & 1.008 & 0.008 & & 1.005 & 0.007 & \\
\hline & & $\theta$ & RSS & 0.998 & 0.002 & 4.000 & 1.001 & 0.002 & 4.000 & 0.998 & 0.002 & 3.500 \\
\hline & & & SRS & 0.542 & 0.070 & & 0.536 & 0.069 & & 0.542 & 0.070 & \\
\hline & & $\alpha$ & RSS & 0.509 & 0.010 & 7.000 & 0.504 & 0.010 & 6.900 & 0.509 & 0.010 & 7.000 \\
\hline & 15 & $\widehat{\theta}$ & SRS & 1.004 & 0.005 & & 1.006 & 0.005 & & 1.003 & 0.005 & \\
\hline & & $\theta$ & RSS & 1.000 & 0.001 & 5.000 & 1.002 & 0.001 & 5.000 & 1.000 & 0.001 & 5.000 \\
\hline
\end{tabular}

TABLE 7: Es, MSE, and Eff values using the MLE, MPS, and OLS methods for the GQLD model parameters with $\alpha=0.8, \theta=1.5$ under SRS and RSS.

\begin{tabular}{|c|c|c|c|c|c|c|c|c|c|c|c|c|}
\hline \multirow{2}{*}{$n$} & \multirow{2}{*}{$k$} & & \multirow{2}{*}{ Method } & \multicolumn{3}{|c|}{ MLE } & \multicolumn{3}{|c|}{ MPS } & \multicolumn{3}{|c|}{ OLS } \\
\hline & & & & Es & MSE & Eff & Es & MSE & Eff & Es & MSE & Eff \\
\hline \multirow{12}{*}{3} & \multirow{4}{*}{5} & \multirow{2}{*}{$\widehat{\alpha}$} & SRS & 2.358 & 5.294 & \multirow{2}{*}{2.322} & 2.416 & 6.034 & \multirow{2}{*}{2.155} & 2.404 & 5.459 & \multirow{2}{*}{2.319} \\
\hline & & & RSS & 1.936 & 2.280 & & 1.960 & 2.800 & & 1.957 & 2.354 & \\
\hline & & \multirow{2}{*}{$\widehat{\theta}$} & SRS & 0.829 & 0.022 & \multirow{2}{*}{2.444} & 0.861 & 0.028 & \multirow{2}{*}{2.154} & 0.823 & 0.025 & \multirow{2}{*}{2.500} \\
\hline & & & RSS & 0.811 & 0.009 & & 0.844 & 0.013 & & 0.804 & 0.010 & \\
\hline & & \multirow{2}{*}{$\widehat{\alpha}$} & SRS & 1.965 & 2.551 & \multirow{2}{*}{8.022} & 1.981 & 2.845 & \multirow{2}{*}{6.379} & 1.957 & 2.499 & \multirow{2}{*}{7.000} \\
\hline & & & RSS & 1.599 & 0.318 & & 1.592 & 0.446 & & 1.615 & 0.357 & \\
\hline & 10 & \multirow{2}{*}{$\widehat{\theta}$} & SRS & 0.814 & 0.009 & \multirow{2}{*}{4.500} & 0.832 & 0.011 & \multirow{2}{*}{2.750} & 0.807 & 0.011 & \multirow{2}{*}{3.667} \\
\hline & & & RSS & 0.803 & 0.002 & & 0.823 & 0.004 & & 0.800 & 0.003 & \\
\hline & \multirow{4}{*}{15} & & SRS & 1.719 & 1.132 & \multirow{2}{*}{9.593} & 1.723 & 1.265 & \multirow{2}{*}{8.490} & 1.737 & 1.210 & \multirow{2}{*}{9.237} \\
\hline & & $\alpha$ & RSS & 1.546 & 0.118 & & 1.533 & 0.149 & & 1.554 & 0.131 & \\
\hline & & \multirow{2}{*}{$\widehat{\theta}$} & SRS & 0.805 & 0.005 & \multirow{2}{*}{5.000} & 0.819 & 0.006 & \multirow{2}{*}{3.000} & 0.801 & 0.007 & \multirow{2}{*}{7.000} \\
\hline & & & RSS & 0.802 & 0.001 & & 0.817 & 0.002 & & 0.800 & 0.001 & \\
\hline
\end{tabular}


TABle 7: Continued.

\begin{tabular}{|c|c|c|c|c|c|c|c|c|c|c|c|c|}
\hline \multirow{2}{*}{$n$} & \multirow{2}{*}{$k$} & & \multirow{2}{*}{ Method } & \multicolumn{3}{|c|}{ MLE } & \multicolumn{3}{|c|}{ MPS } & \multicolumn{3}{|c|}{ OLS } \\
\hline & & & & Es & MSE & Eff & Es & MSE & Eff & Es & MSE & Eff \\
\hline \multirow{12}{*}{4} & \multirow{4}{*}{5} & \multirow{2}{*}{$\widehat{\alpha}$} & SRS & 2.093 & 3.818 & \multirow{2}{*}{1.969} & 2.139 & 4.357 & \multirow{2}{*}{1.824} & 2.125 & 3.870 & \multirow{2}{*}{1.842} \\
\hline & & & RSS & 1.866 & 1.939 & & 1.893 & 2.389 & & 1.904 & 2.101 & \\
\hline & & \multirow{2}{*}{$\widehat{\theta}$} & SRS & 0.813 & 0.015 & \multirow{2}{*}{1.875} & 0.840 & 0.018 & \multirow{2}{*}{1.800} & 0.808 & 0.019 & \multirow{2}{*}{2.111} \\
\hline & & & RSS & 0.809 & 0.008 & & 0.836 & 0.010 & & 0.804 & 0.009 & \\
\hline & \multirow{4}{*}{10} & 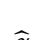 & SRS & 1.818 & 1.581 & \multirow{2}{*}{6.615} & 1.834 & 1.814 & \multirow{2}{*}{5.871} & 1.847 & 1.732 & \multirow{2}{*}{6.439} \\
\hline & & $\alpha$ & RSS & 1.575 & 0.239 & & 1.571 & 0.309 & & 1.588 & 0.269 & \\
\hline & & \multirow{2}{*}{$\widehat{\theta}$} & SRS & 0.810 & 0.006 & \multirow{2}{*}{3.000} & 0.825 & 0.007 & \multirow{2}{*}{2.333} & 0.805 & 0.008 & \multirow{2}{*}{4.000} \\
\hline & & & RSS & 0.803 & 0.002 & & 0.820 & 0.003 & & 0.800 & 0.002 & \\
\hline & \multirow{4}{*}{15} & $\widehat{-}$ & SRS & 1.732 & 0.879 & \multirow{2}{*}{8.969} & 1.737 & 0.981 & \multirow{2}{*}{8.107} & 1.752 & 0.978 & \multirow{2}{*}{9.140} \\
\hline & & $\alpha$ & RSS & 1.534 & 0.098 & & 1.520 & 0.121 & & 1.544 & 0.107 & \\
\hline & & \multirow{2}{*}{$\widehat{\theta}$} & SRS & 0.808 & 0.004 & & 0.820 & 0.005 & & 0.806 & 0.005 & \\
\hline & & & RSS & 0.801 & 0.001 & 4.000 & 0.812 & 0.001 & 5.000 & 0.800 & 0.001 & 5.000 \\
\hline & & & SRS & 1.984 & 2.788 & & 2.013 & 3.207 & & 1.991 & 2.844 & \\
\hline & & $\widehat{\alpha}$ & RSS & 1.761 & 1.192 & 2.339 & 1.812 & 1.679 & 1.910 & 1.788 & 1.262 & 2.254 \\
\hline & 5 & $\widehat{a}$ & SRS & 0.812 & 0.011 & & 0.833 & 0.013 & & 0.805 & 0.013 & \\
\hline & & $\theta$ & RSS & 0.806 & 0.006 & 1.833 & 0.831 & 0.008 & 1.625 & 0.803 & 0.007 & 1.857 \\
\hline & & & SRS & 1.700 & 1.103 & & 1.699 & 1.197 & & 1.706 & 1.098 & \\
\hline & & $\widehat{\alpha}$ & RSS & 1.563 & 0.190 & 5.805 & 1.561 & 0.234 & 5.115 & 1.568 & 0.203 & 5.409 \\
\hline 5 & 10 & & SRS & 0.803 & 0.005 & & 0.816 & 0.006 & & 0.799 & 0.006 & \\
\hline & & $\theta$ & RSS & 0.802 & 0.002 & 2.500 & 0.817 & 0.002 & 3.000 & 0.800 & 0.002 & 3.000 \\
\hline & & & SRS & 1.695 & 0.753 & & 1.697 & 0.815 & & 1.705 & 0.770 & \\
\hline & & $\widehat{\alpha}$ & RSS & 1.528 & 0.082 & 9.183 & 1.523 & 0.099 & 8.232 & 1.533 & 0.088 & 8.750 \\
\hline & 15 & $\widehat{\theta}$ & SRS & 0.805 & 0.004 & & 0.814 & 0.004 & & 0.804 & 0.005 & \\
\hline & & $\theta$ & RSS & 0.801 & 0.001 & 4.000 & 0.811 & 0.001 & 4.000 & 0.800 & 0.001 & 5.000 \\
\hline
\end{tabular}

TABLE 8: Es, MSE, and Eff values using the WLS, CV, and AD methods for the GQLD model parameters with $\alpha=0.8, \theta=1.5$ under SRS and RSS.

\begin{tabular}{|c|c|c|c|c|c|c|c|c|c|c|c|c|}
\hline \multirow{2}{*}{$n$} & \multirow{2}{*}{$k$} & & \multirow{2}{*}{ Method } & \multicolumn{3}{|c|}{ WLS } & \multicolumn{3}{|c|}{$\mathrm{CV}$} & \multicolumn{3}{|c|}{$\mathrm{AD}$} \\
\hline & & & & Es & MSE & Eff & Es & MSE & Eff & Es & MSE & Eff \\
\hline \multirow{12}{*}{3} & \multirow{4}{*}{5} & \multirow{2}{*}{$\widehat{\alpha}$} & SRS & 2.391 & 5.397 & \multirow{2}{*}{2.435} & 2.391 & 5.488 & \multirow{2}{*}{2.387} & 2.368 & 5.233 & \multirow{2}{*}{2.436} \\
\hline & & & RSS & 1.942 & 2.216 & & 1.945 & 2.299 & & 1.928 & 2.148 & \\
\hline & & \multirow{2}{*}{$\widehat{\theta}$} & SRS & 0.821 & 0.024 & \multirow{2}{*}{2.400} & 0.828 & 0.025 & \multirow{2}{*}{2.500} & 0.820 & 0.023 & \multirow{2}{*}{2.556} \\
\hline & & & RSS & 0.803 & 0.010 & & 0.810 & 0.010 & & 0.803 & 0.009 & \\
\hline & \multirow{4}{*}{10} & $\widehat{\alpha}$ & SRS & 1.953 & 2.445 & \multirow{2}{*}{7.570} & 1.955 & 2.494 & \multirow{2}{*}{7.581} & 1.943 & 2.363 & \multirow{2}{*}{7.550} \\
\hline & & $\alpha$ & RSS & 1.610 & 0.323 & & 1.605 & 0.329 & & 1.601 & 0.313 & \\
\hline & & \multirow{2}{*}{$\widehat{\theta}$} & SRS & 0.807 & 0.010 & \multirow{2}{*}{3.333} & 0.811 & 0.010 & \multirow{2}{*}{3.333} & 0.807 & 0.010 & \multirow{2}{*}{5.000} \\
\hline & & & RSS & 0.800 & 0.003 & & 0.804 & 0.003 & & 0.800 & 0.002 & \\
\hline & \multirow{4}{*}{15} & $\widehat{\alpha}$ & SRS & 1.724 & 1.135 & \multirow{2}{*}{9.538} & 1.722 & 1.151 & \multirow{2}{*}{9.672} & 1.721 & 1.117 & \multirow{2}{*}{9.466} \\
\hline & & $\alpha$ & RSS & 1.550 & 0.119 & & 1.545 & 0.119 & & 1.548 & 0.118 & \\
\hline & & \multirow{2}{*}{$\widehat{\theta}$} & SRS & 0.800 & 0.006 & 6000 & 0.803 & 0.006 & & 0.800 & 0.006 & \\
\hline & & & RSS & 0.799 & 0.001 & 6.000 & 0.803 & 0.001 & 0.000 & 0.800 & 0.001 & 0.000 \\
\hline & & & SRS & 2.110 & 3.811 & 1948 & 2.110 & 3.880 & 1952 & 2.105 & 3.750 & 1982 \\
\hline & 5 & $\alpha$ & RSS & 1.878 & 1.956 & 1.948 & 1.875 & 1.988 & 1.952 & 1.867 & 1.892 & 1.982 \\
\hline & 5 & $\widehat{\theta}$ & SRS & 0.807 & 0.017 & 1880 & 0.813 & 0.018 & & 0.807 & 0.016 & \\
\hline & & & RSS & 0.802 & 0.009 & 1.889 & 0.808 & 0.009 & 2.000 & 0.802 & 0.008 & 2.000 \\
\hline & & & SRS & 1.832 & 1.623 & & 1.832 & 1.652 & & 1.826 & 1.579 & \\
\hline & & $\alpha$ & RSS & 1.579 & 0.241 & 6.734 & 1.574 & 0.242 & 6.826 & 1.578 & 0.237 & 6.662 \\
\hline 4 & 10 & $\widehat{\theta}$ & SRS & 0.805 & 0.007 & & 0.808 & 0.007 & & 0.805 & 0.007 & \\
\hline & & $\theta$ & RSS & 0.800 & 0.002 & 3.500 & 0.803 & 0.002 & 3.500 & 0.800 & 0.002 & 3.500 \\
\hline & & & SRS & 1.737 & 0.897 & & 1.735 & 0.905 & & 1.736 & 0.894 & \\
\hline & & $\alpha$ & RSS & 1.538 & 0.098 & 9.153 & 1.534 & 0.098 & 9.235 & 1.536 & 0.098 & 9.122 \\
\hline & 15 & $\widehat{\theta}$ & SRS & 0.806 & 0.005 & & 0.808 & 0.005 & & 0.806 & 0.005 & \\
\hline & & $\theta$ & RSS & 0.799 & 0.001 & 5.000 & 0.802 & 0.001 & 5.000 & 0.800 & 0.001 & 5.000 \\
\hline
\end{tabular}


TABle 8: Continued.

\begin{tabular}{|c|c|c|c|c|c|c|c|c|c|c|c|c|}
\hline \multirow{2}{*}{$n$} & \multirow{2}{*}{$k$} & & \multirow{2}{*}{ Method } & \multicolumn{3}{|c|}{ WLS } & \multicolumn{3}{|c|}{$\mathrm{CV}$} & \multicolumn{3}{|c|}{$\mathrm{AD}$} \\
\hline & & & & Es & MSE & Eff & Es & MSE & Eff & Es & MSE & Eff \\
\hline \multirow{12}{*}{5} & \multirow{4}{*}{5} & \multirow{2}{*}{$\widehat{\alpha}$} & SRS & 1.984 & 2.778 & \multirow{2}{*}{2.338} & 1.986 & 2.838 & \multirow{2}{*}{2.313} & 1.973 & 2.681 & \multirow{2}{*}{2.335} \\
\hline & & & RSS & 1.776 & 1.188 & & 1.774 & 1.227 & & 1.763 & 1.148 & \\
\hline & & \multirow{2}{*}{$\widehat{\theta}$} & SRS & 0.805 & 0.013 & \multirow{2}{*}{2.167} & 0.810 & 0.013 & \multirow{2}{*}{2.167} & 0.805 & 0.012 & \multirow{2}{*}{2.000} \\
\hline & & & RSS & 0.802 & 0.006 & & 0.807 & 0.006 & & 0.801 & 0.006 & \\
\hline & \multirow{4}{*}{10} & \multirow{2}{*}{$\widehat{\alpha}$} & SRS & 1.702 & 1.078 & \multirow{2}{*}{5.704} & 1.701 & 1.097 & \multirow{2}{*}{5.743} & 1.697 & 1.053 & \multirow{2}{*}{5.571} \\
\hline & & & RSS & 1.564 & 0.189 & & 1.560 & 0.191 & & 1.565 & 0.189 & \\
\hline & & $\widehat{\theta}$ & SRS & 0.799 & 0.006 & \multirow[b]{2}{*}{3.000} & 0.802 & 0.006 & \multirow{2}{*}{3.000} & 0.799 & 0.006 & \multirow{2}{*}{3.000} \\
\hline & & $\theta$ & RSS & 0.800 & 0.002 & & 0.803 & 0.002 & & 0.800 & 0.002 & \\
\hline & \multirow{4}{*}{15} & $\widehat{\alpha}$ & SRS & 1.696 & 0.736 & \multirow{2}{*}{8.867} & 1.695 & 0.746 & \multirow{2}{*}{8.988} & 1.696 & 0.736 & \multirow{2}{*}{8.976} \\
\hline & & $\alpha$ & RSS & 1.531 & 0.083 & & 1.528 & 0.083 & & 1.529 & 0.082 & \\
\hline & & $\widehat{\theta}$ & SRS & 0.804 & 0.004 & \multirow{2}{*}{4.000} & 0.806 & 0.004 & \multirow{2}{*}{4.000} & 0.803 & 0.004 & \multirow{2}{*}{4.000} \\
\hline & & $\theta$ & RSS & 0.799 & 0.001 & & 0.801 & 0.001 & & 0.800 & 0.001 & \\
\hline
\end{tabular}

TABle 9: The goodness-of-fit tests for datasets.

\begin{tabular}{lcc}
\hline Dataset & KS & $p$ value \\
\hline Dataset 1 & 0.0928 & 0.5646 \\
Dataset 2 & 0.12244 & 0.8808 \\
\hline
\end{tabular}

TABLE 10: RSS sample taken from dataset 1 for $n=2$ and $k=6$.

\begin{tabular}{lr}
\hline Cycle 1 & Cycle 2 \\
\hline 1.22 & 0.96 \\
1.46 & 0.72 \\
1.08 & 2.93 \\
1.72 & 1.59 \\
1.96 & 2.02 \\
1.76 & 2.53 \\
\hline
\end{tabular}

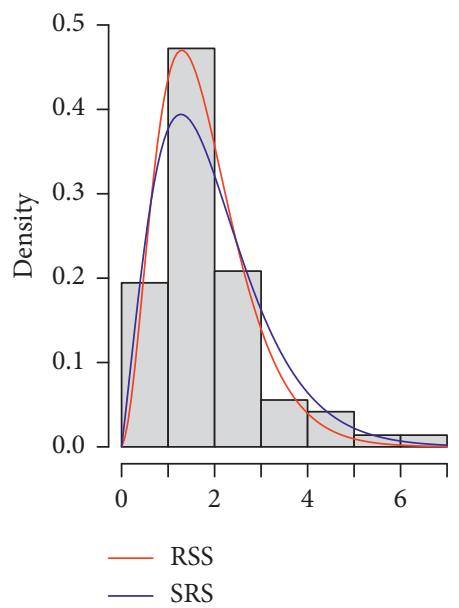

(a)

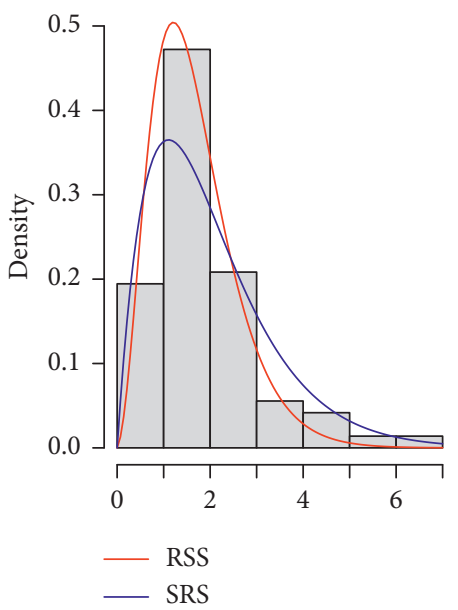

(b)

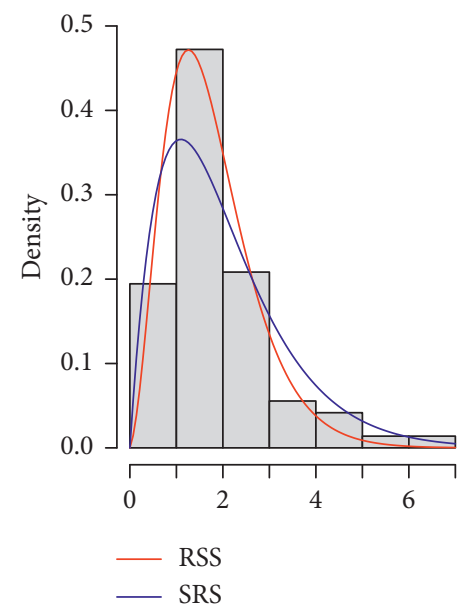

(c)

Figure 1: Continued. 


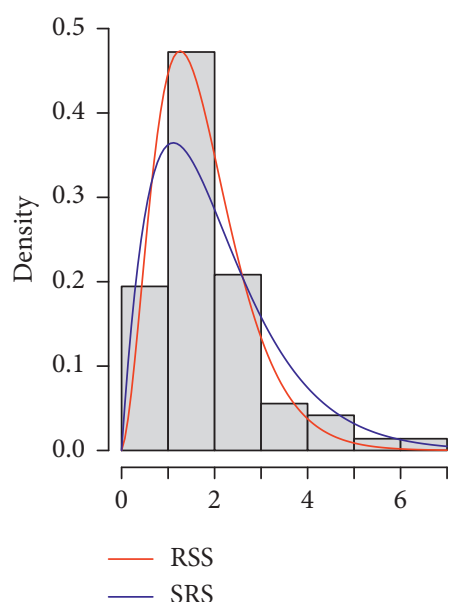

(d)

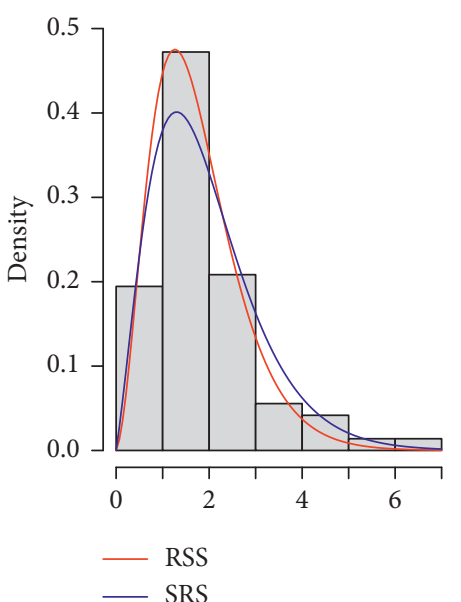

(e)

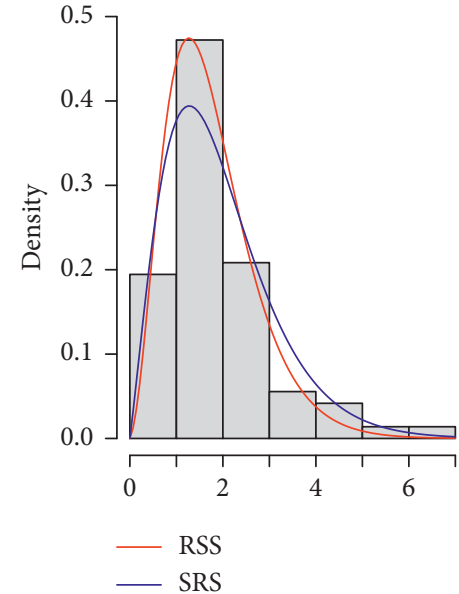

(f)

Figure 1: Plots of estimated probability density functions under RSS and SRS based on dataset 1. (a) MLE. (b) MPS. (c) OLS. (d) WLS. (e) CV. (f) AD.

TABLE 11: The Es, KS, and $p$ value in SRS and RSS designs for dataset 1.

\begin{tabular}{|c|c|c|c|c|c|c|c|c|}
\hline \multirow{2}{*}{ Method } & \multicolumn{2}{|c|}{$\widehat{\alpha}$} & \multicolumn{2}{|c|}{$\widehat{\theta}$} & \multicolumn{2}{|c|}{ KS } & \multicolumn{2}{|c|}{$p$ value } \\
\hline & SRS & RSS & SRS & RSS & SRS & RSS & SRS & RSS \\
\hline MLE & 0.3120 & 0.1701 & 1.8446 & 2.0829 & 0.1048 & 0.0787 & 0.3811 & 0.7341 \\
\hline MPS & 2.4289 & 0.1692 & 1.2911 & 2.2342 & 0.1280 & 0.0834 & 0.1730 & 0.6666 \\
\hline OLS & 2.5625 & 0.2182 & 1.2825 & 2.0813 & 0.1271 & 0.0677 & 0.1793 & 0.8736 \\
\hline WLS & 2.3366 & 0.2132 & 1.2975 & 2.0895 & 0.1287 & 0.0680 & 0.1685 & 0.8705 \\
\hline $\mathrm{CV}$ & 0.5572 & 0.1966 & 1.7033 & 2.1009 & 0.0993 & 0.0658 & 0.4479 & 0.8937 \\
\hline $\mathrm{AD}$ & 0.6852 & 0.1917 & 1.6466 & 2.0988 & 0.1033 & 0.0674 & 0.3994 & 0.8776 \\
\hline
\end{tabular}

TABLE 12: The RSS samples taken from dataset 2 for $n=2$ and $k=4$.

\begin{tabular}{lr}
\hline Cycle 1 & Cycle 22 \\
\hline 41.52 & 17.88 \\
93.12 & 55.56 \\
127.92 & 51.96 \\
98.64 & 127.92 \\
\hline
\end{tabular}

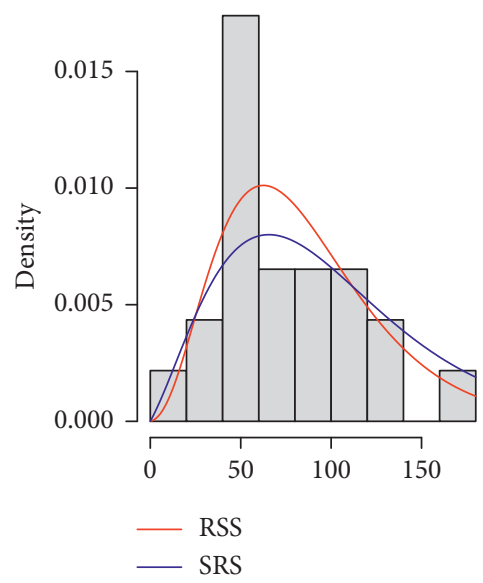

(a)

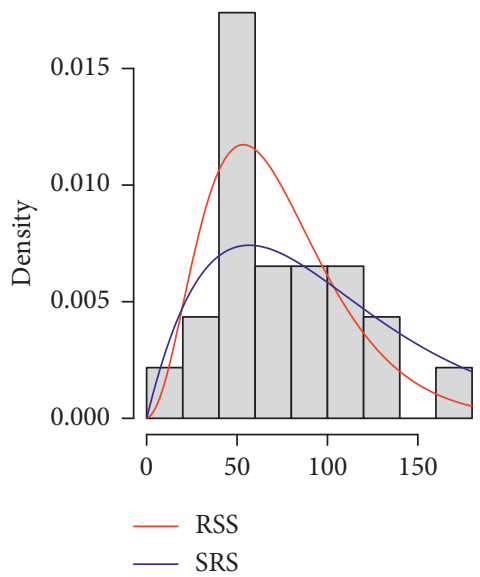

(b)

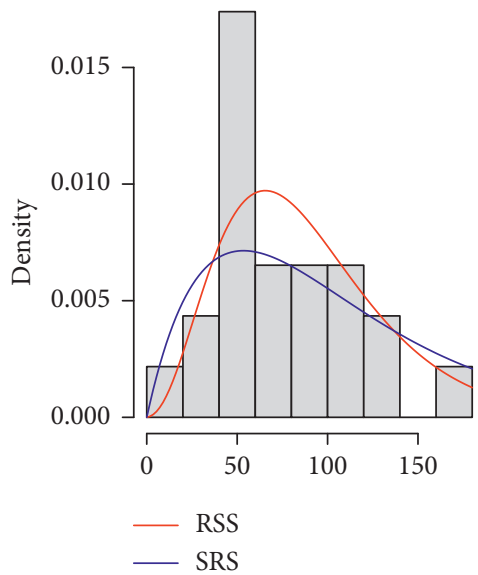

(c)

Figure 2: Continued. 


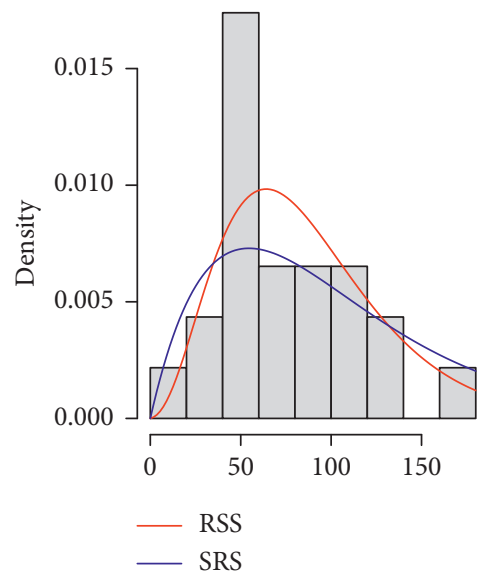

(d)

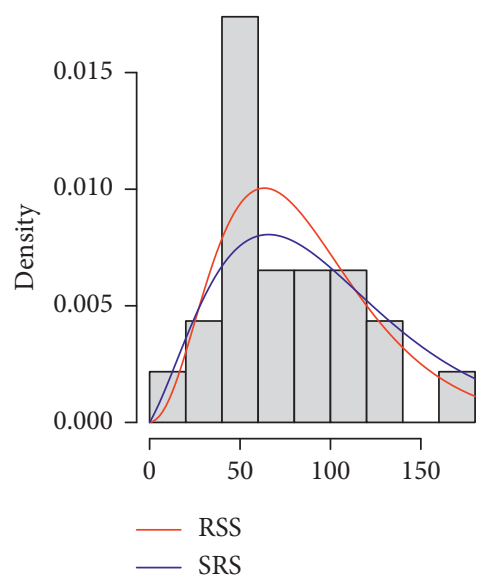

(e)

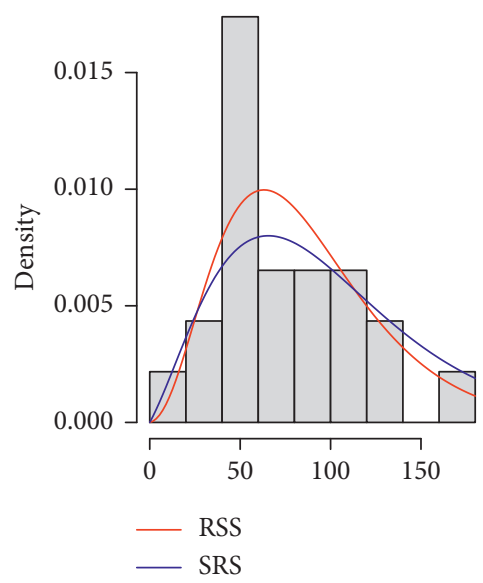

(f)

Figure 2: Plots of the estimated probability density functions under RSS and SRS based on dataset 2. (a) MLE. (b) MPS. (c) OLS. (d) WLS. (e) CV. (f) AD.

TABLE 13: The Es, KS, and $p$ value in SRS and RSS designs for dataset 2.

\begin{tabular}{lccccccrr}
\hline \multirow{2}{*}{ Method } & \multicolumn{2}{c}{$\hat{\alpha}$} & \multicolumn{2}{c}{$\hat{\theta}$} & \multicolumn{2}{c}{ KS } & \multicolumn{2}{c}{$p$ value } \\
& SRS & RSS & SRS & RSS & SRS & RSS & SRS \\
\hline MLE & 0.1100 & 0.0984 & 0.0407 & 0.0450 & 0.2562 & 0.1969 & 0.0809 \\
MPS & 1.8074 & 0.1136 & 0.0275 & 0.0522 & 0.2463 & 0.1024 & 0.1028 \\
OLS & 4.3593 & 0.0865 & 0.0232 & 0.0433 & 0.2615 & 0.2244 & 0.0708 & 0.9494 \\
WLS & 2.7256 & 0.1057 & 0.0253 & 0.0438 & 0.2518 & 0.2100 & 0.0901 & 0.2278 \\
CV & 0.5109 & 0.0851 & 0.0344 & 0.0447 & 0.2474 & 0.2051 & 0.1003 \\
AD & 0.5394 & 0.1063 & 0.0341 & 0.0444 & 0.2473 & 0.2022 & 0.1003 & 0.2516 \\
\hline
\end{tabular}

\section{Conclusion}

In this paper, RSS-based estimation is presented for the GQLD. Six estimation methods are considered, including the maximum likelihood, the maximum product of spacings, ordinary least squares, weighted least squares, Cramer-von Mises, and Anderson-Darling methods. The performances of the proposed estimators are compared with their SRS counterparts using a simulation study and two applications of real data. The numerical simulation results demonstrate that the proposed RSS estimators are better than their SRS counterparts in terms of the MSE for all results presented in the tables based on the same number of measuring units. The results of the real data also confirm the superiority of the RSS design over the SRS design.

For future works, the authors are interested in modifying the GQLD to the transmuted GQLD (see, for example, [46]) and estimating its parameters using the modified robust extreme ranked set sampling [47].

\section{Data Availability}

The real datasets related to guinea pigs and ball bearings used to support the findings of this study are included within the article.

\section{Conflicts of Interest}

The authors declare that they have no conflicts of interest.

\section{Acknowledgments}

The authors thank the Deanship of Scientific Research at King Khalid University for supporting and funding this work through the research group program under grant no. R.G.P. $2 / 82 / 42$.

\section{References}

[1] G. McIntyre, "A method for unbiased selective sampling, using ranked sets," Australian Journal of Agricultural Research, vol. 3, no. 4, pp. 385-390, 1952.

[2] L. S. Halls and T. R. Dell, "Trial of ranked set sampling for forage yields forest," Science, vol. 12, no. 1, pp. 22-26, 1966.

[3] K. Takahasi and K. Wakimoto, "On unbiased estimates of the population mean based on the sample stratified by means of ordering," Annals of the Institute of Statistical Mathematics, vol. 20, no. 1, pp. 1-31, 1968.

[4] T. R. Dell and J. L. Clutter, "Ranked set sampling theory with order statistics background," Biometrics, vol. 28, no. 2, pp. 545-555, 1972.

[5] H. Chen, E. A. Stasny, and D. A. Wolfe, "Ranked set sampling for efficient estimation of a population proportion," Statistics in Medicine, vol. 24, no. 21, pp. 3319-3329, 2005.

[6] P. H. Kvam, "Ranked set sampling based on binary water quality data with covariates," Journal of Agricultural, Biological, and Environmental Statistics, vol. 8, no. 3, pp. 271-279, 2003.

[7] M. Mahdizadeh and E. Zamanzade, "A new reliability measure in ranked set sampling," Statistical Papers, vol. 59, no. 3, pp. 861-891, 2018. 
[8] A. Haq, J. Brown, E. Moltchanova, and A. I. Al-Omari, "Varied L ranked set sampling scheme," Journal of Statistical Theory and Practice, vol. 9, no. 4, pp. 741-767, 2015.

[9] A. Al-Omari and A. Haq, "Goodness-of-fit testing based on new entropy estimator using ranked set sampling and double ranked set sampling for the inverse Gaussian distribution," Environmental Systems Research, vol. 1, no. 1, p. 8, 2012.

[10] A. I. Al-Omari and A. Haq, "Improved quality control charts for monitoring the process mean, using double-ranked set sampling methods," Journal of Applied Statistics, vol. 39, no. 4, pp. 745-763, 2012.

[11] M. F. Al-Saleh and M. A. Al-Kadiri, "Double-ranked set sampling," Statistics \& Probability Letters, vol. 48, no. 2, pp. 205-212, 2000.

[12] H. A. Mutllak, "Median ranked set sampling," Journal of Applied Statistic Science, vol. 6, pp. 245-255, 1997.

[13] E. Zamanzade and A. I. Al-Omari, "New ranked set sampling for estimating the population mean and variance," Hacettepe Journal of Mathematics and Statistics, vol. 45, no. 6, pp. 1891-1905, 2016.

[14] H. M. Samawi, M. S. Ahmed, and W. Abu-Dayyeh, "Estimating the population mean using extreme ranked set sampling," Biometrical Journal, vol. 38, no. 5, pp. 577-586, 1996.

[15] A. D. Al-Nasser, "L ranked set sampling: a generalization procedure for robust visual sampling," Communications in Statistics-Simulation and Computation, vol. 36, no. 1, pp. 33-43, 2007.

[16] A. I. Al-Omari and C. N. Bouza, "Review of ranked set sampling: modifications and applications," Revista Investigacion Operacional, vol. 35, no. 3, pp. 215-240, 2014.

[17] S. A. Al-Hadhrami and A. I. Al-Omari, "Bayesian estimation of the mean of exponential distribution using moving extremes ranked set sampling," Journal of Statistics and Management Systems, vol. 17, no. 4, pp. 365-379, 2014.

[18] A. Haq, J. Brown, E. Moltchanova, and A. I. Al-Omari, "Improved exponentially weighted moving average control charts for monitoring process mean and dispersion," Quality and Reliability Engineering International, vol. 31, no. 2, pp. 217-237, 2015.

[19] A. Santiago, C. N. Bouza, J. M. Sautto, and A. I. Al-Omari, "Randomized response procedure for the estimation of the population ratio using ranked set sampling," Journal of Mathematics and Statistics, vol. 12, no. 2, pp. 107-114, 2016.

[20] A. Haq, J. Brown, E. Moltchanova, and A. I. Al-Omari, "Paired double ranked set sampling," Communications in Statistics-Theory and Methods, vol. 45, no. 1, pp. 2873-2889, 2016.

[21] M. Nassar, A. Z. Afify, S. Dey, and D. Kumar, "A new extension of Weibull distribution: properties and different methods of estimation," Journal of Computational and Applied Mathematics, vol. 336, pp. 439-457, 2018.

[22] M. Nassar, A. Z. Afify, and M. Shakhatreh, "Estimation methods of alpha power exponential distribution with applications to engineering and medical data," Pakistan Journal of Statistics and Operation Research, vol. 16, no. 1, pp. 149-166, 2020.

[23] A. Z. Afifiy and O. A. Mohamed, "A new three-parameter exponential distribution with variable shapes for the hazard rate: estimation and applications," Mathematics, vol. 8, pp. 1-17, 2020.

[24] N. M. Alfaer, A. M. Gemeay, H. M. Aljohani, and A. Z. Afify, "The extended log-logistic distribution: inference and actuarial applications," Mathematics, vol. 9, pp. 1-22, 2021.
[25] A. Z. Afifiy, M. Nassar, G. M. Cordeiro, and D. Kumar, "The Weibull Marshall-Olkin Lindley distribution: properties and estimation," Journal of Taibah University for Science, vol. 14, no. 1, pp. 192-204, 2020.

[26] O. M. Yousef and S. A. Al-Subh, "Estimation of Gumbel parameters under ranked set sampling," Journal of Modern Applied Statistical Methods, vol. 13, no. 2, pp. 432-443, 2014.

[27] M. Hussian, "Bayesian and maximum likelihood estimation for Kumaraswamy distribution based on ranked set sampling," American Journal of Mathematics and Statistics, vol. 4, no. 1, pp. 30-37, 2014.

[28] W. Chen, M. Xie, and M. Wu, "Parametric estimation for the scale parameter for scale distributions using moving extremes ranked set sampling," Statistics \& Probability Letters, vol. 83, no. 9, pp. 2060-2066, 2013.

[29] W. A. Abu-Dayyeh, S. A. Al-Subh, and H. A. Muttlak, "Logistic parameters estimation using simple random sampling and ranked set sampling data," Applied Mathematics and Computation, vol. 150, no. 2, pp. 543-554, 2004.

[30] V. C. Pedroso, C. A. Taconeli, and S. R. Giolo, "Estimation based on ranked set sampling for the two-parameter Birnbaum-Saunders distribution," Journal of Statistical Computation and Simulation, vol. 91, no. 2, pp. 316-333, 2021.

[31] F. G. Akgul, K. Yu, and B. Senoglu, "Estimation of the system reliability for generalized inverse Lindley distribution based on different sampling designs," Communications in StatisticsTheory and Methods, vol. 50, no. 11, pp. 1532-1546, 2021.

[32] C. A. Taconeli and W. H. Bonat, "On the performance of estimation methods under ranked set sampling," Computational Statistics, vol. 35, no. 4, pp. 1805-1826, 2020.

[33] S. L. Stokes, "Parametric ranked set sampling," Annals of the Institute of Statistical Mathematics, vol. 47, pp. 465-482, 1995.

[34] S. Dey, M. Salehi, and J. Ahmadi, "Rayleigh distribution revisited via ranked set sampling," Metron, vol. 75, no. 1, pp. 69-85, 2017.

[35] H. J. Khamnei and S. R. Mayan, "Comparison of parameter estimation in the exponentiated Gumbel distribution based on ranked set sampling and simple random sampling," Journal of Mathematics and Statistical Science, vol. 2016, pp. 490-497, 2016.

[36] M. F. Al-Saleh and S. A. Al-Hadrami, "Parametric estimation for the location parameter for symmetric distributions using moving extremes ranked set sampling with application to trees data," Environmetrics, vol. 14, no. 7, pp. 651-664, 2003.

[37] S. Benchiha and A. I. Al-Omari, "Generalized quasi Lindley distribution: theoretical properties, estimation methods, and applications," Electronic Journal of Applied Statistical Analysis, vol. 14, no. 1, 2020.

[38] R. C. H. Cheng and N. A. K. Amin, "Maximum product-ofspacings estimation with applications to the log-normal distribution," Technical report, Department of Mathematics, University of Wales, Cardiff, UK, 1979.

[39] R. C. H. Cheng and N. A. K. Amin, "Estimating parameters in continuous univariate distributions with a shifted origin," Journal of the Royal Statistical Society: Series B (Methodological), vol. 45, no. 3, pp. 394-403, 1983.

[40] J. J. Swain, S. Venkatraman, and J. R. Wilson, "Least-squares estimation of distribution functions in Johnson's translation system," Journal of Statistical Computation and Simulation, vol. 29, no. 4, pp. 271-297, 1988.

[41] R. B. D'Agostino and M. A. Stephens, Goodness-of-Fit Techniques, Marcel Dekker, New York, NY, USA, 1986. 
[42] A. Luceño, "Fitting the generalized Pareto distribution to data using maximum goodness-of-fit estimators," Computational Statistics \& Data Analysis, vol. 51, no. 2, pp. 904-917, 2006.

[43] T. Bjerkedal, "Acquisition of resistance in Guinea pigs infected with different doses of virulent tubercle Bacilli," American Journal of Epidemiology, vol. 72, no. 1, pp. 130-148, 1960.

[44] A. Z. Afify, H. M. Yousof, G. M. Cordeiro, Z. M. Nofal, and M. Ahmad, "The Kumaraswamy Marshall-Olkin Fréchet distribution with applications," Journal of ISOSS, vol. 2, no. 2, pp. 151-168, 2016.

[45] J. F. Lawless, Statistical Models and Methods for Lifetime Data, John Wiley \& Sons, New York, NY, USA, 1982.

[46] M. Garaibah and A. I. Al-Omari, "Transmuted Ishita distribution and its applications," Journal of Statistics Applications and Probability, vol. 8, no. 2, pp. 67-81, 2019.

[47] A. I. Al-Omari, "Estimation of mean based on modified robust extreme ranked set sampling," Journal of Statistical Computation and Simulation, vol. 81, no. 8, pp. 1055-1066, 2011. 